\title{
Feynman Graphs and Generalized Eikonal Approach to High Energy Knock-Out Processes
}

\author{
L. L. Frankfurt ${ }^{a, c}$, M. M. Sargsian ${ }^{a, d}$, M. I. Strikman ${ }^{b, c}$ \\ (a) School of Physics and Astronomy, Tel Aviv University, Tel Aviv, 69978, Israel \\ (b) Department of Physics, Pennsylvania State University, University Park, PA 16802 \\ (c) Institute for Nuclear Physics, St. Petersburg, Russia \\ (d) Yerevan Physics Institute, Yerevan, 375036, Armenia
}

March 14, 2018

\begin{abstract}
The cross section of hard semi-exclusive $A\left(e, e^{\prime} N\right)(A-1)$ reactions for fixed missing energy and momentum is calculated within the eikonal approximation. Relativistic dynamics and kinematics of high energy processes are unambiguously accounted for by using the analysis of appropriate Feynman diagrams. A significant dependence of the final state interactions on the missing energy is found, which is important for interpretation of forthcoming color transparency experiments. A new, more stringent kinematic restriction on the region where the contribution of short-range nucleon correlations is enhanced in semi-exclusive knock-out processes is derived. It is also demonstrated that the use of light-cone variables leads to a considerable simplification of the description of high-energy knock-out reactions.
\end{abstract}

Typeset using REVTEX 


\section{INTRODUCTION}

With the advance of high energy, high intensity electron facilities (see e.g. [1] 3]) the high momentum transfer semi-exclusive reactions are becoming a practical tool for the investigation of the microscopic structure of nuclei, nuclear matter and the color transparency phenomenon. However, theoretical methods which were successful in medium-energy nuclear physics should be upgraded in order to describe processes where energies transferred to a nuclear target are $\gtrsim f e w G e V$. This paper focuses on the calculation of the influence of the final state interactions (FSI) on high energy hard semi-exclusive $A\left(e, e^{\prime} N\right) X$ reactions, for energies of knocked out nucleon $E_{N} \geq 1 \mathrm{GeV}$ and for states $X$ representing ground or excited states of the residual nucleus.

At energies $E_{N} \leq 1 \mathrm{GeV}$ the final state interactions (FSI) are usually evaluated in terms of interactions of knock-out nucleons with an effective potential of the residual system the optical model approximation, (see, for example, Ref. [4]). Parameters of the effective potential are adjusted to describe data on elastic $N-(A-1)$ scattering for projectile energies close to $E_{N}$. Two important features of high-energy FSI make the extension of the medium energy formalism to high energies problematic. Firstly, the number of essential partial waves increases rapidly with the energy of the $N,(A-1)$ system. Secondly, the $N N$ interaction which is practically elastic for $E_{N} \leq 500 \mathrm{MeV}$ becomes predominantly inelastic for $E_{N}>1 \mathrm{GeV}$. Hence the problem of scattering hardly can be treated as a many body quantum mechanical problem. Introducing in this situation a predominantly imaginary potential to account for hadron production (not only for excitations of residual system as in the case of intermediate energies) is not a well defined mathematical concept. So theoretical methods successful below $1 \mathrm{GeV}$ become ineffective at the energies which can be probed at Jefferson Lab [1], HERMES [2] and ELFE [3].

FSI at higher energies $\left(E_{N}>1 \mathrm{GeV}\right)$ are often described within the approximation of the additivity of phases, acquired in the sequential rescatterings of high-energy projectiles off the target nucleons (nonrelativistic Glauber model [5]). This approximation made it possible to describe the data on elastic $h A$ scattering at hadron energies $1 \mathrm{GeV}<E_{h}<$ $10-15 \mathrm{GeV}$ (cf. Refs. [6,7]). It has been also applied to the description of cross sections of $A\left(e, e^{\prime} N\right)(A-1)$ reactions [8 18] integrated over the excitation energies $\left(E_{\text {exc }}\right)$ of the residual $(A-1)$ nucleon system, for small momentum of the residual system $\vec{p}_{A-1} \leq p_{F}$. In Ref. [19,20] the cross section of $A\left(e, e^{\prime} N\right)(A-1)$ reactions has been calculated for small excitation energies that are characteristic for particular shells of a target nucleus with $A \gtrsim$ $12-16$. Thus the dependence of FSI on missing energy- $E_{m}\left(\sim E_{\text {exc }}\right)$ was not essential in the previous calculations. Furthermore such a dependence is not important for total cross sections, small angle coherent and noncoherent (summed over residual nuclear excitations) scatterings in $h A$ reactions. However the dependence of FSI on the missing energy is part of the color transparency phenomena in high-energy quasielastic processes where restrictions on the missing energy should be imposed to suppress inelastic processes where pions are produced [21,22]. It is also important in the studying of short-range nucleon correlations in nuclei in semi-exclusive reactions, where large value of missing energy should be ensured.

In this paper we consider high-energy semi-exclusive $A\left(e, e^{\prime} N\right)(A-1)$ reactions, where both missing momentum and missing energy are fixed. We investigate the implications of the nonzero value of the missing energy on the FSI of the knocked-out nucleon. 
The linear increase with incident energy of the coherence length of strong interactions leads to a change of the underlying physical picture of hadron-nucleus scattering - from sequential rescatterings to coherent interactions with all nucleons at a given impact parameter. First evidence for that was obtained by Mandelstam [23] who analyzed planar Feynman diagrams corresponding to rescattering diagrams of the nonrelativistic Glauber approximation. He found that the contribution of these diagrams tends to zero in the high-energy limit.

Later on Gribov [24] developed a quantitative theory of high-energy $h A$ interactions and demonstrated that the small value of the ratio of inelastic diffractive and elastic cross sections serves as a small parameter, justifying Glauber-type formulae. We restrict the analysis in the paper to the range of energies where inelastic diffraction in the soft hadron processes is a small correction (i.e. energies of the knocked-out nucleon $\lesssim 10 \mathrm{GeV}$ ). We also restricted by the photon virtualities $Q^{2} \sim 1-3 \mathrm{GeV}^{2}$, where color coherent phenomena are expected to be a small correction. At larger $Q^{2}$ the deduced formulae can be used as a baseline model for searching for color coherent phenomena. (Note that the small ratio of inelastic and elastic diffraction reflects small dispersion of strengths of interaction for soft processes. These fluctuations are naturally much larger in the case of hard processes where a probe selects a rare, small size configurations in the struck nucleon. For a review of physics of the color coherence phenomena see Refs. [25,26]).

At large $Q^{2}$ and small struck nucleon momenta it is safe to neglect the dependence of the $e N$ scattering amplitude on nucleon binding since the energy scale of the hard interaction is much larger than the nuclear energy scale. At the same time, when missing momenta and missing energies relevant for knock-out processes are comparable with the Fermi momenta and the nucleon binding energy, it is necessary to take them into account in the calculation of the nuclear part of the scattering amplitude. Obviously, this can not be done unambiguously within the optical model and the Glauber type approximations, which neglect nucleon Fermi momenta in the nuclei. To calculate FSI of the knocked out nucleon we derive the formulae of the eikonal approximation which account for the nucleon Fermi motion. Our derivation is based on the analysis of the Feynman diagrams corresponding to the $A\left(e, e^{\prime} N\right) X$ reaction.

The method of the derivation of the formulae of the eikonal approximation on the basis of the analysis of the relevant Feynman diagrams has been suggested long ago for hadronnucleus collisions in Refs. [27,28]. It has been shown in Refs. [27,28] under what conditions the Feynman diagram description of the hadron-nucleus scattering processes leads to the optical model or the Glauber type approximation. The main advantage of the Feynman graph approach is that it takes into account the relativistic kinematics of high-energy processes. Particularly it accounts for an important feature of high energy small-angle elastic (diffractive) scatterings - the conservation of the light-cone momentum $p_{-} \equiv \sqrt{m^{2}+p^{2}}-p_{z}$, where $p_{z}$ is the component of nucleon Fermi momentum $\vec{p}$ in the projectile momentum direction. In the present paper we apply this method to calculate FSI in $A\left(e, e^{\prime} N\right)(A-1)$ reactions and extend the results of ref. [29] for ${ }^{2} H\left(e, e^{\prime}\right)(p n)$ process to the case of nucleon knock-out processes off ${ }^{3} \mathrm{He}\left({ }^{3} \mathrm{H}\right)$ (eq.(33)). After deriving formulae of the impulse approximation, single and double rescattering terms, we generalize the obtained results to the case of a nucleus with arbitrary $A$. In the case of a deuteron target we found significant effects of the missing energy [29] (Fig.3). We demonstrate that even larger effects are expected for knock out of nucleons off ${ }^{3} \mathrm{He},{ }^{4} \mathrm{He}$ targets.

It follows from the formulae derived in the paper that when missing momenta and energy 
are not negligible in knock-out reactions, the optical model approximation becomes unreliable. Also, we found in this kinematics significant corrections to the conventional Glauber type formulae (Fig.4).

Based on the analysis of the derived formulae we determine optimal kinematic conditions for the investigation of the short-range nucleon correlations in nuclei in semi-inclusive reactions. In particular, if one wants to use for such investigations kinematics $x_{B j}>1$ it is necessary to impose additional conditions on the recoil energy of the residual system (eq.(40)). Such conditions allow to suppress the contribution of low-momentum component of the nuclear wave function due to FSI (Section 4).

We demonstrate also that light-cone kinematics of high-energy knock-out reaction is naturally accounted for if nucleon Fermi momenta in the nucleus are parameterized in terms of the light-cone variables.

\section{SCATTERING AMPLITUDE}

In this section we consider the scattering amplitude for a knocked-out nucleon to undergo $n$ rescatterings off the nucleons of $(A-1)$ residual system. The case $n=0$ corresponds to the impulse approximation (IA) in which the knocked out nucleon does not interact with residual nucleus. We systematically neglect in this paper the diffractive excitation of the nucleons in the intermediate states. In soft QCD processes this is a small correction for the knock-out nucleon energies $\lesssim 10 \mathrm{GeV}$. In the hard processes (that is when $Q^{2}$ - virtuality of the photon is sufficiently large $\left.\left(\gtrsim 6-8 G e V^{2}\right)\right)$ such an approximation can not be justified even within this energy range, see for example discussion in Ref. [25]. However our aim is to perform calculations in the kinematics where CT phenomenon is still a small correction.

The scattering amplitude can be represented by covariant Feynman diagrams of Fig.1, in the approximation when only elastic rescatterings are accounted for, as:

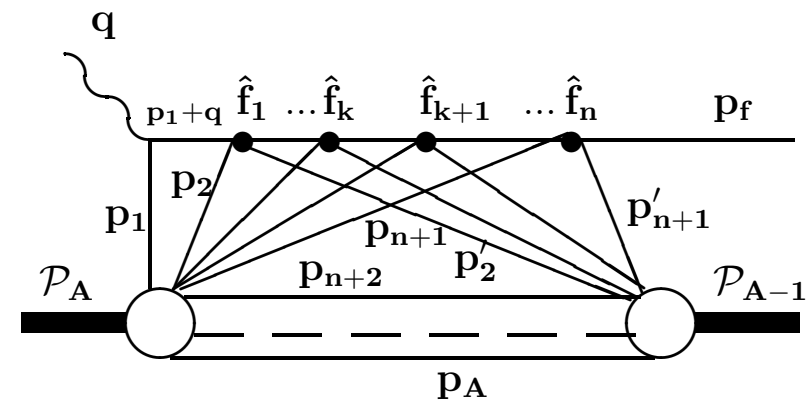

Fig. 1 n-fold rescattering diagram.

$$
\begin{aligned}
& F_{A, A-1}^{(n)}\left(q, p_{f}\right)=\sum_{h} \frac{1}{n !(A-n-1) !} \prod_{i=1}^{A} \prod_{j=2}^{A} \int d^{4} p_{i} d^{4} p_{j}^{\prime} \frac{1}{\left[i(2 \pi)^{4}\right]^{n+1}} \\
& \delta^{4}\left(\sum_{i=1}^{A} p_{i}-\mathcal{P}_{A}\right) \delta^{4}\left(\sum_{j=2}^{A} p_{j}^{\prime}-\mathcal{P}_{A-1}\right) \prod_{m=n+2}^{A} \delta^{4}\left(p_{m}-p_{m}^{\prime}\right) \times \\
& \frac{\Gamma_{A}\left(p_{1}, \ldots, p_{A}\right)}{D\left(p_{1}\right) D\left(p_{2}\right) . . D\left(p_{n+1}\right) D\left(p_{n+2}\right) . . D\left(p_{A}\right)} \frac{F_{h}^{e m}\left(Q^{2}\right)}{D\left(p_{1}+q\right)} \frac{f_{1}^{N N}\left(p_{2}, p_{2}^{\prime}\right) . . f_{n}^{N N}\left(p_{n+1}, p_{n+1}^{\prime}\right)}{D\left(l_{1}\right) . . D\left(l_{k}\right) . . D\left(l_{n-1}\right)} \times
\end{aligned}
$$




$$
\frac{\Gamma_{A-1}\left(p_{2}^{\prime}, . p_{n+1}^{\prime}, . p_{n+2} . ., p_{A}\right)}{D\left(p_{2}^{\prime}\right) . . D\left(p_{n+1}^{\prime}\right)}
$$

where, for the sake of simplicity, we neglected the spin dependent effects. Here $\mathcal{P}_{A}$ and $\mathcal{P}_{A-1}$ are the four momenta of target nucleus, and final $(A-1)$ system, $p_{j}$ and $p_{j}^{\prime}$ are nucleon momenta in the nucleus $A$ and residual $(A-1)$ system respectively. $\sum_{h}$ in eq.(1) goes over virtual photon interactions with different nucleons, where $F_{h}^{e m}\left(Q^{2}\right)$ is electromagnetic vertices. $-D\left(p_{k}\right)^{-1}=\left(p_{k}^{2}-m^{2}+i \epsilon\right)^{-1}$ is the propagator of a nucleon with momentum $p_{k}$. The $f_{k}^{N N}\left(p_{k+1}, p_{k+1}^{\prime}\right)$ is the amplitude of $N N$ scattering, $\frac{d \sigma}{d t} \sim \frac{|f|^{2}}{s_{k}^{2}}$, where $s_{k}$ is the total invariant energy of two interacting nucleons. $-D\left(l_{k}\right)^{-1}$ is the propagator of the struck nucleon in the intermediate state, with momentum $l_{k}=q+p_{1}+\sum_{i=2}^{k}\left(p_{i}-p_{i}^{\prime}\right)$ between $k-1$-th and $k$-th rescatterings. The factor $n !(A-n-1)$ ! accounts for the combinatorics of $n$ - rescatterings and $(A-n-1)$ spectator nucleons. Following Ref. [27] we choose the "minus" sign for the nucleon propagators to simplify the calculation of the overall sign of the scattering amplitude - for each closed contour one gets the factor $\frac{1}{i(2 \pi)^{4}}$ with no additional sign.

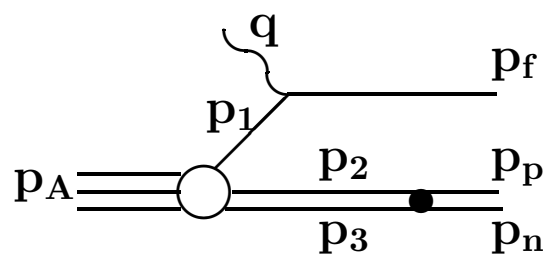

(a)

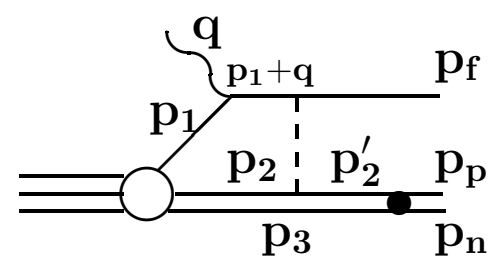

(b)

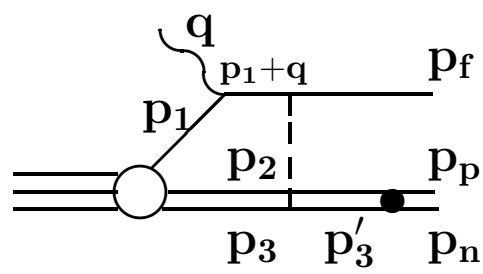

(c)

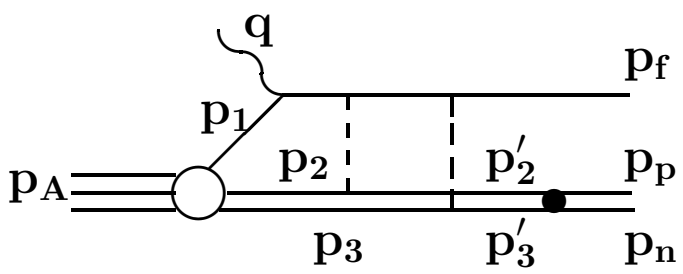

(d)

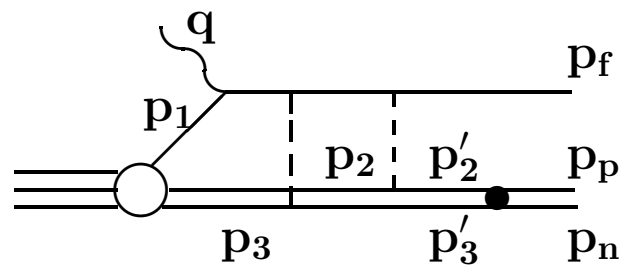

(e)

Fig. 2 Feynman diagrams corresponding to ${ }^{3} H e\left(e, e^{\prime} p\right) p n$ scattering. Dashed lines represent effective $N N$ scattering, full circle represents the residual interaction between spectator nucleons.

The vertex functions $\Gamma_{A}\left(p_{1}, \ldots, p_{A}\right)$ and $\Gamma_{A-1}\left(p_{2}^{\prime}, \ldots, p_{A}\right)$ describe transitions of 
"nucleus $A^{\prime \prime}$ to "A nucleons" with momenta $\left\{p_{n}\right\}$ and transitions of " $(A-1)$ nucleons" with momenta $\left\{p_{n}^{\prime}\right\}$ to " $(A-1)$ nucleon final state" respectively. The intermediate spectator state in the diagram of Fig. 1 is expressed in terms of nucleons because the closure over various nuclear excitations in the intermediate state is used (for the details see Appendix A).

After evaluation of the intermediate state nucleon propagators the covariant amplitude will be reduced to a set of time ordered noncovariant diagrams. This will help to establish the correspondence between the vertex functions and the nuclear wave functions. We derive formulae for the impulse approximation and first two rescattering terms (i.e. single and double rescattering). To simplify derivations we consider $\left(e, e^{\prime} N\right)$ reactions off a three nucleon system (see Fig.2) and then generalize obtained results to an arbitrary $A$.

\section{IMPULSE APPROXIMATION}

First, we consider the $A\left(e, e^{\prime} N\right)(A-1)$ reaction, where the final state consists of a noninteracting energetic nucleon $N$ and a $(A-1)$ residual state which can be either the nuclear bound state or break up system of $(A-1)$ nucleons. For the scattering off a three nucleon system this reaction corresponds to the covariant diagram of Fig.2a, which is the $n=0$ term in the scattering amplitude of eq.(1). For the $n=0$ term of eq.(11), performing the integrations over $\delta$-functions due to the energy-momentum conservation we obtain:

$$
F^{(a)} \equiv F_{A, A-1}^{(0)}\left(q, p_{f}\right)=\int d^{4} p_{3} \frac{\Gamma_{A}\left(p_{1}, p_{2}, p_{3}\right) F_{1}^{e m}\left(Q^{2}\right) \Gamma\left(p_{2}, p_{3}\right)}{D\left(p_{1}\right) D\left(p_{2}\right) D\left(p_{3}\right)},
$$

where

$$
\begin{aligned}
& p_{1}=\mathcal{P}_{A}-\mathcal{P}_{A-1}, \\
& p_{2}+p_{3}=\mathcal{P}_{A-1} .
\end{aligned}
$$

Here $\Gamma_{A}$ and $\Gamma_{A-1}$ correspond to the nuclear vertices represented in Fig.2, by empty and full circles respectively. In eqs.(2) and (3) $p_{1}$ is the momentum of the interacting nucleon and $p_{2}, p_{3}$ are the momenta of spectator nucleons. To simplify the derivation we neglect the antisymmetrization of the initial and final nucleon states, which can be easily accounted for through the corresponding wave functions (see below).

The scattering amplitude $F^{(a)}$ is Lorenz invariant and corresponds to the sum of the non-covariant diagrams with different time orderings between nuclear $\Gamma_{A}, \Gamma_{A-1}$ and electromagnetic vertices $F_{h}^{e m}$. The impulse approximation corresponds to the time ordered noncovariant diagram of Fig.2a, where virtual photon is absorbed by a target nucleon which does not interact in the final state. Other time orderings correspond to vacuum fluctuations.

We will perform calculation in the nucleus rest frame in the kinematics where Fermi momenta of target nucleons are not large. Hence we will restrict the consideration to the range of missing momenta $p_{m}$ and missing energies $E_{m}$ :

$$
\begin{aligned}
\left|\vec{p}_{m}\right| & \equiv\left|\vec{p}_{f}-\vec{q}\right| \lesssim 400 \mathrm{MeV} / \mathrm{c} \\
\alpha & \equiv \frac{E_{f}-p_{f z}}{m}-\frac{q_{o}-|\vec{q}|}{m} \approx \frac{m-E_{m}-p_{m z}}{m} \approx 1 \pm 0.3,
\end{aligned}
$$


where $\vec{p}_{f}$ is the momentum of final knocked-out nucleon, $q_{0}$ and $\vec{q}$ are the energy and momentum of virtual photon. The $z$ axis is chosen in the $\vec{q}$ direction. The direction transverse to the $\vec{q}$ would be labeled by $t . E_{m} \equiv E_{A-1}+m-E_{A} \cdot \alpha$ is the light-cone fraction of the momentum of the target carried by interacting nucleon scaled to vary between 0 and $A$.

If we restrict by the kinematics defined in eq.(1) then in the set of the noncovariant diagrams, comprising the covariant diagram of Fig.2a, one can neglect the diagrams which correspond to the vacuum fluctuations, (see e.g. Refs. [30]). The latter become increasingly important at larger Fermi momenta of the target nucleons. An effective method to account for the diagrams with vacuum fluctuations is light-cone approach [30 34] where for some components of the electromagnetic current ("good" components) their contribution is suppressed and scattering amplitude has the form rather similar to the conventional impulse approximation. This physics, being interesting by itself, is beyond of the scope of this paper.

Overall in the discussed kinematics (eq.(14)) the relativistic effects in the nuclear wave function are a small correction [30,29] and the impulse approximation can be calculated via nonrelativistic reduction of the covariant nuclear vertices in eq.(2). Such a reduction corresponds to taking the residue over $d p_{3}^{0}$, at the nearest nucleon pole in the spectator nucleon propagator $D\left(p_{3}\right)^{-1}$. Thus we neglect nonnucleon degrees of freedom in a nucleus. The restriction by the nearest pole in the nucleon propagators follows from the observation that in the considered kinematics (四) where nuclear excitations are small as compared to the scale of energies characteristic for the nucleon excitations, this is the only pole not corresponding to $N \bar{N}$ production. Neglect of discontinuities related to the thresholds of pion production is justified in QCD because, for a small pion momenta, the pions are Goldstone bosons of spontaneously broken chiral symmetry (see the discussion in Ref. [31]).

Taking residue over the spectator nucleon propagator effectively corresponds to the replacement

$$
\int \frac{d p_{3}^{0}}{2 \pi i} \frac{1}{D\left(p_{3}\right)} \rightarrow \frac{1}{E_{3}} \approx \frac{1}{2 m}
$$

After this integration one is left with the time ordered diagram corresponding to the IA, where virtual photon knocks-out the target nucleon with momentum $p_{1}$, leaving the residual $A-1$ nucleus with a particular excitation energy $E_{e x c}=E_{m}-\frac{p_{m}^{2}}{M_{A-1}}-\left|\epsilon_{A}\right|$ where $\epsilon_{A}-$ is the binding energy of the target nucleus. Nonrelativistic reduction allows to define the momentum space wave function through the vertex function as (c.f. Refs. [27,28]):

$$
\psi_{A}\left(p_{1}, p_{2}, \ldots p_{A}\right)=\frac{1}{\left(\sqrt{(2 \pi)^{3} 2 m}\right)^{A-1}} \frac{\Gamma_{A}\left(p_{1}, p_{2}, \ldots p_{A}\right)}{D\left(p_{1}\right)}
$$

where wave functions are normalized as: $\int\left|\psi_{A}\left(p_{1}, p_{2}, \ldots p_{A}\right)\right|^{2} d^{3} p_{1} d^{2} p_{2} . . d^{3} p_{A}=1$. We define the wave function of the final (residual nucleus + knocked-out nucleon) state as $\psi_{A-1} / \sqrt{2 m}$, where $\psi_{A-1}$ defined according to eq. (5), with $A$ replaced by $A-1$ and the additional factor $1 / \sqrt{2 m}$ accounts for the normalization of the knocked-out nucleon wave function. With these definitions eq.(2) obtains the form of the conventional IA expression:

$$
T^{(a)}=\sqrt{(2 \pi)^{3}}(2 \pi)^{3} \int d^{3} p_{3} \psi_{A}\left(p_{m}, p_{2}, p_{3}\right) F_{1}^{e m}\left(Q^{2}\right) \psi_{A-1}^{+}\left(p_{2}, p_{3}\right),
$$

where $\vec{p}_{m}=\vec{p}_{f}-\vec{q}$ - is the measured missing momentum, and $\vec{p}_{2}=-\vec{p}_{3}-\vec{p}_{m}$. The spin and isospin indices and antisymmetrization of wave functions are implicit in eq.(6). 
Introducing the coordinate space wave functions for $\psi_{A}$ and $\psi_{A-1}$ as:

$$
\psi_{j}\left(p_{1}, p_{2}, \ldots p_{j}\right)=\left(\frac{1}{\sqrt{(2 \pi)^{3}}}\right)^{j} \int d^{3} x_{1} d^{3} x_{2}, \ldots d^{3} x_{j} e^{-i\left(\vec{x}_{1} \cdot \vec{p}_{1}+\vec{x}_{2} \cdot \vec{p}_{2}+\ldots+\vec{x}_{j} \cdot \vec{p}_{j}\right)} \phi_{A}\left(x_{1}, x_{2}, \ldots x_{j}\right),
$$

where $j \equiv A, A-1$ we can represent the IA amplitude as follows:

$$
\begin{aligned}
T^{(a)}= & \int d^{3} x_{1} d^{3} x_{2} d^{3} x_{3} \phi_{A}\left(x_{1}, x_{2}, x_{3}\right) F_{1}^{e m}\left(Q^{2}\right) e^{i \vec{x}_{1} \cdot \vec{q}} \phi_{A-1}^{\dagger}\left(x_{2}, x_{3}\right) e^{-i \vec{x}_{1} \cdot \vec{p}_{f}}= \\
& \int d^{3} x_{1} d^{3} x_{2} d^{3} x_{3} \phi_{A}\left(x_{1}, x_{2}, x_{3}\right) F_{1}^{e m}\left(Q^{2}\right) e^{-i \frac{3}{2} \vec{p}_{m} \cdot \vec{x}_{1}} \phi_{A-1}^{\dagger}\left(x_{2}-x_{3}\right) .
\end{aligned}
$$

We define $x_{1}$, as the coordinate of the struck nucleon, and $x_{2}, x_{3}$ as coordinates of the residual nuclear system. In the last part of eq.(8) we introduce the wave function of residual nuclear system with separated internal and center of mass (CM) motion by:

$$
\phi_{A-1}\left(y_{2}, y_{3}\right)=\phi\left(y_{2}-y_{3}\right) e^{i \frac{\vec{y}_{2}+\vec{y}_{3}}{2} \cdot \vec{p}_{c m}}
$$

with $\vec{p}_{c m}$ is CM momentum of residual two-nucleon system.

Obviously, as follows from eqs.(6,8), within the impulse approximation measuring $q$ and $p_{f}$ one directly measures the Fermi momentum of a nucleon in the nucleus: $\vec{p}_{1}=\vec{p}_{m}=\vec{p}_{f}-\vec{q}$.

\section{SINGLE RESCATTERING AMPLITUDE}

The diagrams of Fig.2b and Fig.2c describe the processes where the struck (fast) nucleon rescatters off one of the spectator nucleons. The general expression for the amplitude corresponding to the diagram Fig. $2 \mathrm{~b}$ is given by $n=1$ term of eq.(1) as:

$$
T^{(b)}=\int \frac{\Gamma\left(p_{1}, p_{2}, p_{3}\right)}{D\left(p_{1}\right) D\left(p_{2}\right) D\left(p_{3}\right)} F_{1}^{e m}\left(Q^{2}\right) \frac{f^{N N}\left(p_{2}^{\prime}-p_{2}\right)}{D\left(p_{1}+q\right)} \frac{\Gamma\left(p_{2}^{\prime}, p_{3}\right)}{D\left(p_{2}^{\prime}\right)} \frac{d^{4} p_{2}}{i(2 \pi)^{4}} \frac{d^{4} p_{3}}{i(2 \pi)^{4}}
$$

where

$$
p_{1}=\mathcal{P}_{A}-p_{2}-p_{3} ; \quad p_{2}^{\prime}=\mathcal{P}_{A-1}-p_{3} .
$$

Our interest is in the kinematics where contribution of the vacuum diagram is negligible, thus as in the previous section, we can perform the integration over the $d^{0} p_{2} d^{0} p_{3}$ by taking residues over the poles in the nucleon propagators $D\left(p_{2}\right)^{-1}$ and $D\left(p_{3}\right)^{-1}$. The integration results in the replacement

$$
\int \frac{d p_{2,3}^{0}}{2 \pi i} \frac{1}{D\left(p_{2,3}\right)} \rightarrow \frac{1}{2 E_{2,3}} \approx \frac{1}{2 m}
$$

After the integrations over $d^{0} p_{2} d^{0} p_{3}$ are performed, the diagram of Fig.2b becomes the noncovariant time ordered diagram, where a virtual photon is absorbed by the target nucleon, and then the produced fast nucleon rescatters off a spectator nucleon:

$$
T^{(b)}=\frac{1}{\sqrt{2 m} \cdot(2 m)^{2}} \int \frac{\Gamma\left(p_{1}, p_{2}, p_{3}\right)}{D\left(p_{1}\right)} F_{1}^{e m}\left(Q^{2}\right) \frac{f^{N N}\left(p_{2}^{\prime}-p_{2}\right)}{D\left(p_{1}+q\right)} \frac{\Gamma\left(p_{2}^{\prime}, p_{3}\right)}{D\left(p_{2}^{\prime}\right)} \frac{d^{3} p_{2}}{(2 \pi)^{3}} \frac{d^{3} p_{3}}{(2 \pi)^{3}}
$$


The definition of the momentum space wave functions is now straightforward. It corresponds to the nonrelativistic reduction of the nuclear vertices $\Gamma_{A}$ and $\Gamma_{A-1}$ as given by eq.(5). Hence we obtain:

$$
T^{(b)}=\frac{\sqrt{(2 \pi)^{3}}(2 \pi)^{3}}{2 m} \int \psi_{A}\left(p_{1}, p_{2}, p_{3}\right) F_{1}^{e m}\left(Q^{2}\right) \frac{f^{N N}\left(p_{2}^{\prime}-p_{2}\right)}{D\left(p_{1}+q\right)} \psi_{A-1}\left(p_{2}^{\prime}, p_{3}\right) \frac{d^{3} p_{1}}{(2 \pi)^{3}} \frac{d^{3} p_{3}}{(2 \pi)^{3}} .
$$

Here $D\left(p_{1}+q\right)^{-1}$ describes the struck nucleon propagator before the rescattering:

$$
\begin{aligned}
-D\left(p_{1}+q\right) & =\left(p_{1}+q\right)^{2}-m^{2}+i \epsilon=p_{1}^{2}+2 p_{1} q+q^{2}-m^{2}+i \epsilon \approx \\
& \approx 2 q\left[\frac{2 m q_{0}-Q^{2}}{2 q}-p_{1 z}+i \epsilon\right],
\end{aligned}
$$

where $q \equiv|\vec{q}|$ and because of nonrelativistic approximation for nuclear wave function (see Appendix A) we neglect $\frac{p_{1}^{2}}{2 m^{2}}$ as compared to 1 . The factor $\frac{2 m q_{0}-Q^{2}}{2 q}$ is fixed by the external kinematics, since both $q_{0}$ and $Q^{2}$ are measured. It follows from $\left(q+p_{A}-p_{A-1}\right)^{2}=m^{2}$ that:

$$
\frac{2 m q_{0}-Q^{2}}{2 q}=p_{z}^{m}+\frac{q_{0}}{q}\left(m+E_{A-1}-M_{A}\right)+\frac{m^{2}-\tilde{m}^{2}}{2 q} \approx p_{z}^{m}+\Delta_{0}
$$

where $p_{z}^{m}=p_{f z}-q, \tilde{m}^{2} \equiv\left[p_{A}-p_{A-1}\right]^{2}$ is the virtuality of the interacting nucleon, and

$$
\Delta_{0}=\frac{q_{0}}{q}\left(m+E_{A-1}-M_{A}\right) \equiv \frac{q_{0}}{q} E_{m}
$$

where $E_{m}=q_{0}+m-\sqrt{m^{2}+p_{f}^{2}}$ is the missing energy in the reaction. In the case of the three body breakup kinematics for the scattering off the ${ }^{3} H e$ target $E_{m}=T_{p n}+\left|\epsilon_{b}\right|$, where $T_{p n}$ is the kinetic energy of the spectator (two nucleon) system and $\left|\epsilon_{b}\right|$ - is the modulus of the target binding energy. In the right-hand side of eq.(15) we neglected the term $\frac{m^{2}-\tilde{m}^{2}}{2 q}$ related to the virtuality of interacting nucleon since at fixed recoil energy this factor is of the order of $O\left(E_{m} / q\right)$ and its contribution decreases with increase of the transferred momentum $q$.

Inserting eq.(15) into the expression for the propagator of knocked-out nucleon(14) and redefining the $N N$ scattering amplitude as $f^{N N} / 2 q m=f^{N N}$, to be in accordance with the optical theorem in the form: $\operatorname{Im} f^{N N}(t=0)=\sigma_{\text {tot }}^{N N}$, we obtain:

$$
\begin{array}{r}
T^{(b)}=-\frac{\sqrt{(2 \pi)^{3}}(2 \pi)^{3}}{2} \int \psi_{A}\left(p_{1}, p_{2}, p_{3}\right) F_{1}^{e m}\left(Q^{2}\right) \frac{f^{N N}\left(p_{2}^{\prime}-p_{2}\right)}{\left[p_{z}^{m}+\Delta_{0}-p_{1 z}+i \epsilon\right]} \psi_{A-1}\left(p_{2}^{\prime}, p_{3}\right) \\
\frac{d^{3} p_{1}}{(2 \pi)^{3}} \frac{d^{3} p_{3}}{(2 \pi)^{3}} .
\end{array}
$$

We can perform integration over $p_{1 z}$ by transforming integrals into the coordinate space representation and using the fact that for the soft $N N$ scatterings, at high energies, $f^{N N}\left(p_{2}^{\prime}-\right.$ $\left.p_{2}\right) \approx f^{N N}\left(p_{2 t}^{\prime}-p_{2 t}\right)$. Using the coordinate space representation of the nuclear wave functions given by eq.(7) and the coordinate space representation of the nucleon propagators

$$
\frac{1}{\left[p_{z}^{m}+\Delta_{0}-p_{1 z}+i \epsilon\right]}=-i \int \Theta\left(z^{0}\right) e^{i\left(p_{m}^{z}+\Delta_{0}-p_{1 z}\right) z^{0}} d z^{0}
$$


we obtain for the single rescattering amplitude $F^{(b)}$ (see Appendix B):

$$
\begin{aligned}
T^{(b)}= & \frac{i}{2} \int d^{3} x_{1} d^{3} x_{2} d^{3} x_{3} \phi_{A}\left(x_{1}, x_{2}, x_{3}\right) \Theta\left(z_{2}-z_{1}\right) e^{i\left(\vec{b}_{2}-\vec{b}_{1}\right) \cdot \vec{k}_{t}} F_{1}^{e m}\left(Q^{2}\right) \\
& f^{N N}\left(p_{2 t}^{\prime}-p_{2 t}\right) e^{i \Delta_{0}\left(z_{2}-z_{1}\right)} e^{-i \frac{3}{2} \vec{p}_{m} \cdot \vec{x}_{1}} \phi^{\dagger}\left(x_{2}-x_{3}\right) \frac{d^{2} k}{(2 \pi)^{2}}
\end{aligned}
$$

where $\vec{k}_{t}=\vec{p}_{1}^{t}-\vec{p}_{m}^{t}=\vec{p}^{\prime}{ }_{2 t}-\vec{p}_{2 t}$ is the momentum transferred in the rescattering and $\vec{b}_{1}$, $\vec{b}_{2}$ are transverse components of the vectors $\vec{x}_{1}$, and $\vec{x}_{2}$. It is convenient to introduce the generalized profile function [29]:

$$
\Gamma^{N N}(x, \Delta)=\frac{1}{2 i} e^{i \Delta z} \int f^{N N}\left(k_{t}\right) e^{i \vec{b} \cdot \vec{k}_{t}} \frac{d^{2} k_{t}}{(2 \pi)^{2}} .
$$

Using $\Gamma^{N N}(x, \Delta)$, we can write eq.(19) in a form resembling the Glauber theory expression for single rescattering:

$$
\begin{aligned}
& T^{(b)}=-\int d^{3} x_{1} d^{3} x_{2} d^{3} x_{3} \phi_{A}\left(x_{1}, x_{2}, x_{3}\right) F_{1}^{e m}\left(Q^{2}\right) \Theta\left(z_{2}-z_{1}\right) \Gamma^{N N}\left(x_{2}-x_{1}, \Delta_{0}\right) \\
& e^{-i \frac{3}{2} \vec{p}_{m} \cdot \vec{x}_{1}} \phi^{\dagger}\left(x_{2}-x_{3}\right) .
\end{aligned}
$$

The amplitude for the single rescattering - $T^{(c)}$, corresponding to Fig.2c, can be obtained from eq.(21) replacing $r_{2} \leftrightarrow r_{3}$. Thus the whole amplitude, which includes IA and the single rescattering contributions is:

$$
\begin{array}{r}
T^{(a)}+T^{(b)}+T^{(c)}=\int d^{3} x_{1} d^{3} x_{2} d^{3} x_{3} \phi_{A}\left(x_{1}, x_{2}, x_{3}\right) F_{1}^{e m}\left(Q^{2}\right)\left[1+\hat{T}_{F S I}^{(1)}\right] \times \\
e^{-i \frac{3}{2} \vec{p}_{m} \cdot \vec{x}_{1}} \phi^{\dagger}\left(x_{2}-x_{3}\right),
\end{array}
$$

where

$$
\hat{T}_{F S I}^{(1)}=\Theta\left(z_{2}-z_{1}\right) \Gamma^{N N}\left(x_{2}-x_{1}, \Delta_{0}\right)+\Theta\left(z_{3}-z_{1}\right) \Gamma^{N N}\left(x_{3}-x_{1}, \Delta_{0}\right)
$$

is the operator of FSI corresponding to the single rescattering contribution. Eq.(23) can be generalized for the scattering off a nucleus with atomic number $A$ as follows:

$$
\hat{T}^{F S I(1)}=1+\sum_{j=2}^{A} \Theta\left(z_{j}-z_{1}\right) \Gamma^{N N}\left(x_{1}-x_{j}, \Delta_{0}\right) .
$$

The deduced operator for FSI have the form analogous to the familiar operator deduced within nonrelativistic Glauber approximation [5]. The key difference is that the profile function - $\Gamma$ is modified by the additional phase factor $e^{i \Delta z}$. This factor accounts for the geometry of high-energy processes related to the longitudinal momentum transfer in the rescattering. Note that similar factor is present in the expressions for the diffractive photoproduction of vector mesons [6, 35], where it accounts for the difference between the masses of final vector mesons and initial (virtual) photon $\left(\left|t_{\text {min }}\right|>0\right)$. In this case it reflects finite longitudinal distances $\left(\leq R_{A}\right)$ for photoproduction at intermediate energies. In our case the factor $\Delta_{0}$ arises from excitations in the residual nuclear system (see eq.(16)). 


\section{FIGURES}

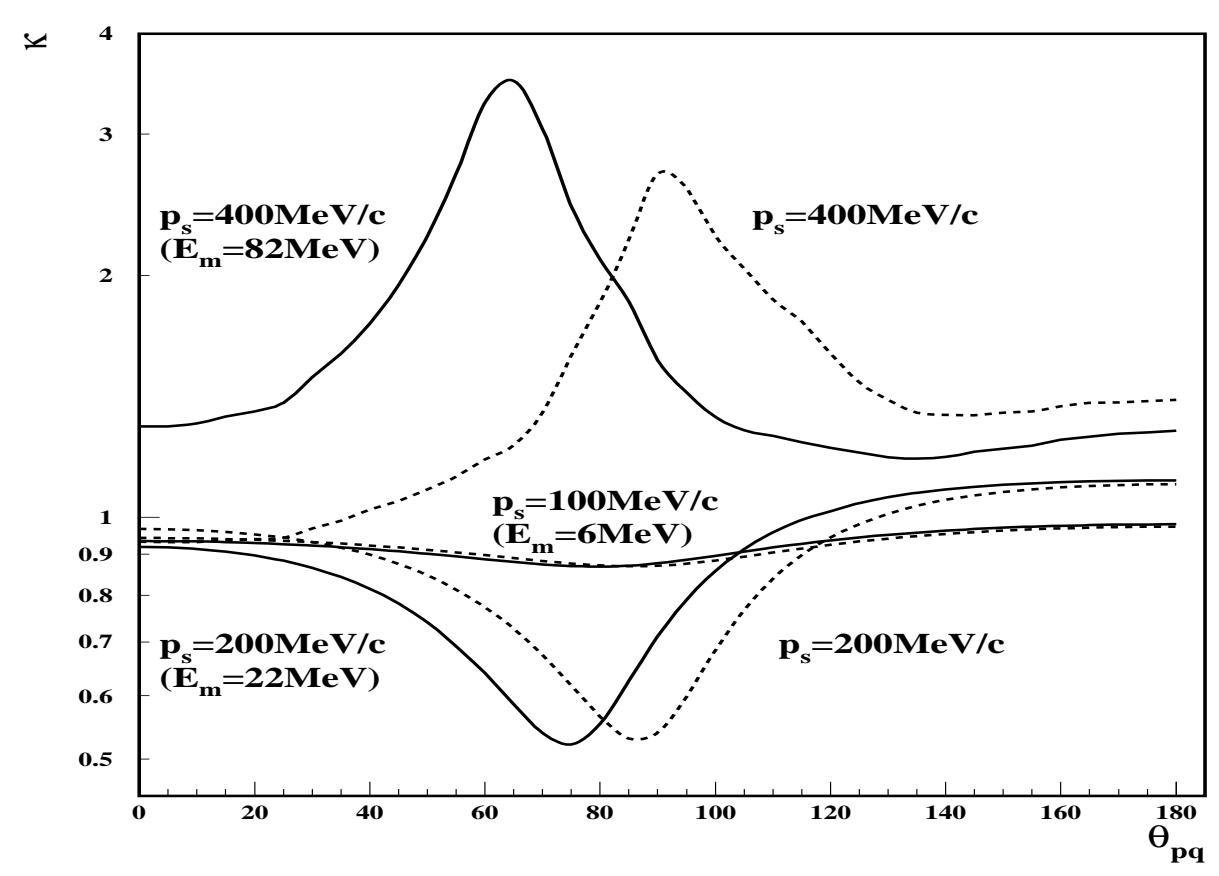

Fig. 3 Dependence of the ratio $\kappa$, of the $d\left(e, e^{\prime} p\right) n$ cross section calculated including the $I A$ and FSI terms to the cross section which includes IA term only, on the angle $\Theta_{\vec{p}_{s} \vec{q}} \equiv$ $\overrightarrow{p_{s}} \vec{q}$ for different spectator momenta $p_{s}$. Solid line corresponds to FSI, calculated according to eq.(17]24), dashed line corresponds to FSI calculated according to conventional Glauber approximation.

To illustrate the importance of derived modification of scattering operator we calculate the cross section of $\left(e, e^{\prime} N\right)$ scattering off the deuteron target where eq.(24) provides the complete form for the FSI operator [29]. Fig.3 represents the ratio of the full cross section to the cross section calculated within the impulse approximation. For small momenta of a target nucleon $\left(p_{s}<100 \mathrm{MeV} / \mathrm{c}\right)$ or for small excitation energies $\left(E_{m} \approx \frac{p_{s}^{2}}{2 m}\right)$ predictions of generalized eikonal approximation (solid line) and conventional Glauber approximation (dashed line) coincidences. This demonstrates the consistency of our approach with Glauber approximation where target nucleons are interpreted as a stationary scatterers and their Fermi momenta has been neglected. However at larger Fermi momenta (or excitation energies) predictions of both approaches are considerably different. For example for $p_{s}=400 \mathrm{MeV} / \mathrm{c}$ the prediction for angular dependence of the maximal contribution from the rescattering amplitude (i.e. the position of the maximum in Fig.3) differs as much as by $30^{\circ}$. Such a difference is quite dramatic and can be checked in the forthcoming experiments at Jefferson Laboratory [36, 37]. Practically the same difference arises for example in the break up of ${ }^{3} \mathrm{He}$ if one of spectators has momentum $p \sim 0$ [37. 


\section{DOUBLE RESCATTERING AMPLITUDE}

The diagrams of Fig.2d,2e describe the amplitude of the process where struck nucleon rescatters sequentially off both spectator nucleons. From eq.(1) choosing $n=2$, for the double rescattering amplitude of Fig.2d, we obtain:

$$
\begin{aligned}
& T^{(d)}= \\
& \int \frac{\Gamma\left(p_{1}, p_{2}, p_{3}\right)}{D\left(p_{1}\right) D\left(p_{2}\right) D\left(p_{3}\right)} F_{1}^{e m}\left(Q^{2}\right) \frac{f^{N N}\left(p_{2}^{\prime}-p_{2}\right)}{D\left(p_{1}+q\right)} \frac{f^{N N}\left(p_{3}^{\prime}-p_{3}\right)}{D\left(p_{1}+q+p_{2}-p_{2}^{\prime}\right)} \\
& \frac{\Gamma\left(p_{2}^{\prime}, p_{3}^{\prime}\right)}{D\left(p_{2}^{\prime}\right) D\left(p_{3}^{\prime}\right)} \delta^{4}\left(p_{A}-p_{2}-p_{3}-p_{1}\right) \delta^{4}\left(p_{A-1}-p_{2}^{\prime}-p_{3}^{\prime}\right) d^{4} p_{1} d^{4} p_{2} d^{4} p_{3} d^{4} p_{2}^{\prime} d^{4} p_{3}^{\prime}\left[\frac{1}{i(2 \pi)^{4}}\right]^{3}= \\
& \int \frac{\Gamma\left(p_{1}, p_{2}, p_{3}\right)}{D\left(p_{1}\right) D\left(p_{2}\right) D\left(p_{3}\right)} F_{1}^{e m}\left(Q^{2}\right) \frac{f^{N N}\left(p_{2}^{\prime}-p_{2}\right)}{D\left(p_{1}+q\right)} \frac{f^{N N}\left(p_{3}^{\prime}-p_{3}\right)}{D\left(p_{1}+q+p_{2}-p_{2}^{\prime}\right)} \times \\
& \frac{\Gamma\left(p_{2}^{\prime}, p_{3}^{\prime}\right)}{D\left(p_{2}^{\prime}\right) D\left(p_{3}^{\prime}\right)} \frac{d^{4} p_{2}}{i(2 \pi)^{4}} \frac{d^{4} p_{3}}{i(2 \pi)^{4}} \frac{d^{4} p_{3}^{\prime}}{i(2 \pi)^{4}},
\end{aligned}
$$

where

$$
p_{1}=\mathcal{P}_{A}-p_{3}-p_{2} ; \quad p_{2}^{\prime}=\mathcal{P}_{A-1}-p_{3}^{\prime}
$$

Then, using the same approximations as for the cases of IA and single rescattering amplitudes we can perform integration over $d^{0} p_{2}, d^{0} p_{3}, d^{0} p_{3}^{\prime}$, which effectively results in the replacement $\int \frac{d^{0} p_{j}}{2 \pi i D\left(p_{j}\right)} \rightarrow \frac{1}{2 E_{j}} \approx \frac{1}{2 m},\left(j=2,3,3^{\prime}\right)$.

Using eq.(26) and the definition of the initial and final state wave functions from Section IIII we obtain:

$$
\begin{aligned}
T^{(d)}= & \frac{\sqrt{(2 \pi)^{3}}(2 \pi)^{3}}{4 m^{2}} \int \psi_{A}\left(p_{1}, p_{2}, p_{3}\right) F_{1}^{e m}\left(Q^{2}\right) \frac{f^{N N}\left(p_{2}^{\prime}-p_{2}\right)}{D\left(p_{1}+q\right)} \frac{f^{N N}\left(p_{3}^{\prime}-p_{3}\right)}{D\left(p_{1}+q+p_{2}-p_{2}^{\prime}\right)} \times \\
& \psi_{A-1}\left(p_{2}^{\prime}, p_{3}^{\prime}\right) \frac{d^{3} p_{1}}{(2 \pi)^{3}} \frac{d^{3} p_{3}}{(2 \pi)^{3}} \frac{d^{3} p_{3}^{\prime}}{(2 \pi)^{3}}
\end{aligned}
$$

where $D\left(p_{1}+q\right)$ is given by eq.(14). Using eq.(26) we can rewrite $D\left(p_{1}+q+p_{2}-p_{2}^{\prime}\right)$ as:

$$
\begin{aligned}
-D\left(p_{1}+q+p_{2}-p_{2}^{\prime}\right)= & -D\left(q+p_{A}-p_{A-1}+p_{3}^{\prime}-p_{3}\right) \\
= & \left(q+p_{A}-p_{A-1}+p_{3}^{\prime}-p_{3}\right)^{2}-m^{2}+i \epsilon \approx \\
& 2 q\left[\frac{q_{0}}{q}\left(E_{3}^{\prime}-E_{3}\right)-\left(p_{3 z}^{\prime}-p_{3 z}\right)+i \epsilon\right]=\left[\left(\Delta_{3}-\left(p_{3 z}^{\prime}-p_{3 z}\right)+i \epsilon\right] .\right.
\end{aligned}
$$

In the derivation of eq. 28) we use the kinematic condition for the quasielastic scattering: $\left(q+p_{A}-p_{A-1}\right)^{2}=m^{2}$ and define $\Delta_{3}=\frac{q_{0}}{q}\left(E_{3}^{\prime}-E_{3}\right)$. Similar to the previous section after redefining the $N N$ amplitude as $f^{N N} / 2 q m \rightarrow f^{N N}$ we obtain:

$$
\begin{aligned}
T^{(d)=} & \frac{\sqrt{(2 \pi)^{3}}(2 \pi)^{3}}{4} \int \psi_{A}\left(p_{1}, p_{2}, p_{3}\right) F_{1}^{e m}\left(Q^{2}\right) \times \\
& \frac{f^{N N}\left(p_{2}^{\prime}-p_{2}\right)}{p_{z}^{m}+\Delta_{0}-p_{1 z}+i \epsilon} \cdot \frac{f^{N N}\left(p_{3}^{\prime}-p_{3}\right)}{\Delta_{3}-\left(p_{3 z}^{\prime}-p_{3 z}\right)+i \epsilon} \psi_{A-1}\left(p_{2}^{\prime}, p_{3}^{\prime}\right) \frac{d^{3} p_{1}}{(2 \pi)^{3}} \frac{d^{3} p_{3}}{(2 \pi)^{3}} \frac{d^{3} p_{3}^{\prime}}{(2 \pi)^{3}} .
\end{aligned}
$$


Integration in eq.(29) can be performed in the coordinate space, using the Fourier transformation of the wave functions according to eq.(7) and nucleon propagators according to eq.(18). For the double rescattering amplitude, $T^{(b)}$, we obtain (see Appendix B):

$$
\begin{aligned}
T^{(d)} & =\frac{i^{2}}{4} \int d^{3} x_{1} d^{3} x_{2} d^{3} x_{3} \phi_{A}\left(x_{1}, x_{2}, x_{3}\right) F_{1}^{e m}\left(Q^{2}\right) \\
& \times\left[\Theta\left(z_{2}-z_{1}\right) f^{N N}\left(k_{2 t}\right) e^{i \vec{k}_{2 t} \cdot\left(\vec{b}_{2}-\vec{b}_{1}\right)} e^{i\left(\Delta_{0}-\Delta_{3}\right)\left(z_{2}-z_{1}\right)} \frac{d^{2} k_{2}}{(2 \pi)^{2}}\right] \\
& \times\left[\Theta\left(z_{3}-z_{2}\right) f^{N N}\left(k_{3 t}\right) e^{i \vec{k}_{3 t} \cdot\left(\vec{b}_{3}-\vec{b}_{1}\right)} e^{i \Delta_{3}\left(z_{3}-z_{1}\right)} \frac{d^{2} k_{3}}{(2 \pi)^{2}}\right] e^{-i \frac{3}{2} \vec{x}_{1} \cdot \vec{p}_{m}} \phi^{\dagger}\left(x_{2}-x_{3}\right),
\end{aligned}
$$

where $k_{2 t}$ and $k_{3 t}$ are the momenta transferred in the first and second rescattering vertices in Fig.2d.

For a complete calculation of the double rescattering term one should take into account the amplitude $F^{(e)}$ too, which corresponds to Fig.2e. This amplitude can be derived from eq.(30) by interchanging coordinates of nucleons " 2 " and "3". Finally, using definition of the modified profile functions from eq.(20) we obtain for $T^{(d)}+T^{(e)}$ :

$$
\begin{aligned}
\hat{T}^{(2)} \equiv T^{(d)}+T^{(e)}= & \int d^{3} x_{1} d^{3} x_{2} d^{3} x_{3} \phi_{A}\left(x_{1}, x_{2}, x_{3}\right) F_{1}^{e m}\left(Q^{2}\right) \mathcal{O}^{(2)}\left(z_{1}, z_{2}, z_{3}, \Delta_{0}, \Delta_{2}, \Delta_{3}\right) \\
& \Gamma^{N N}\left(x_{2}-x_{1}, \Delta_{0}\right) \Gamma^{N N}\left(x_{3}-x_{1}, \Delta_{0}\right) e^{-i \frac{3}{2} \vec{x}_{1} \cdot \vec{p}_{m}} \phi^{\dagger}\left(x_{2}-x_{3}\right) .
\end{aligned}
$$

Here $\hat{T}_{F S I}^{(2)}$ - is the operator of FSI describing the double rescattering contribution and we introduce the $\mathcal{O}$ function which accounts for the geometry of two sequential rescatterings as:

$$
\begin{aligned}
\mathcal{O}^{(2)}\left(z_{1}, z_{2}, z_{3}, \Delta_{0}, \Delta_{2}, \Delta_{3}\right)= & \\
& \Theta\left(z_{2}-z_{1}\right) \Theta\left(z_{3}-z_{2}\right) e^{-i \Delta_{3}\left(z_{2}-z_{1}\right)} e^{i\left(\Delta_{3}-\Delta_{0}\right)\left(z_{3}-z_{1}\right)} \\
& +\Theta\left(z_{3}-z_{1}\right) \Theta\left(z_{2}-z_{3}\right) e^{-i \Delta_{2}\left(z_{3}-z_{1}\right)} e^{i\left(\Delta_{2}-\Delta_{0}\right)\left(z_{2}-z_{1}\right)} .
\end{aligned}
$$

Eqs.(8, 21,22, 31) represent the complete set of scattering amplitudes necessary to calculate knock-out reactions off the ${ }^{3} \mathrm{He}\left({ }^{3} \mathrm{H}\right)$ target:

$$
\begin{array}{r}
T^{(a)}+T^{(b)}+T^{(c)}+T^{(d)}+T^{(e)}=\int d^{3} x_{1} d^{3} x_{2} d^{3} x_{3} \phi_{A}\left(x_{1}, x_{2}, x_{3}\right) F_{1}^{e m}\left(Q^{2}\right) \\
{\left[1+\hat{T}_{F S I}^{(1)}+\hat{T}_{F S I}^{(2)}\right] e^{-i \frac{3}{2} \vec{p}_{m} \cdot \vec{x}_{1}} \phi^{\dagger}\left(x_{2}-x_{3}\right) .}
\end{array}
$$

It is worth to note that in the derivation of above formulae no specific assumptions have been made on the nuclear wave functions. Therefore realistic wave functions of nuclei can be implemented to calculate the high-energy knock-out reactions for different configurations of the residual two-nucleon system.

Eq.(31) can be generalized to calculate the double rescattering amplitude for $\left(e, e^{\prime} N\right)$ reactions off $A$ nucleus as follows:

$$
\hat{T}_{F S I}^{(2)}=\sum_{i, j=2 ; i \neq j}^{A} \mathcal{O}^{(2)}\left(z_{1}, z_{i}, z_{j}, \Delta_{0}, \Delta_{i}, \Delta_{j}\right) \Gamma^{N N}\left(x_{i}-x_{1}, \Delta_{0}\right) \Gamma^{N N}\left(x_{j}-x_{1}, \Delta_{0}\right) .
$$


Generalization of the FSI operator $\hat{T}_{F S I}^{(2)}$ to multiple rescatterings is straightforward:

$$
\begin{gathered}
\hat{T}_{F S I}^{(n)}=\sum_{i, j, . . n=2 ; i \neq j \neq . . n}^{A} \mathcal{O}^{(n)}\left(z_{1}, z_{i}, z_{j}, \ldots z_{n}, \Delta_{0}, \Delta_{i}, \Delta_{j} \ldots \Delta_{n}\right) \times \\
\Gamma^{N N}\left(x_{i}-x_{1}, \Delta_{0}\right) \cdot \Gamma^{N N}\left(x_{j}-x_{1}, \Delta_{0}\right) \cdot \ldots \cdot \Gamma^{N N}\left(x_{n}-x_{1}, \Delta_{0}\right),
\end{gathered}
$$

where

$$
\begin{array}{r}
\mathcal{O}^{(n)}\left(z_{1}, z_{i}, z_{j}, \ldots, z_{n} \Delta_{0}, \Delta_{i}, \Delta_{j} \ldots \Delta_{n}\right)=\sum_{p e r m} \Theta\left(z_{i}-z_{1}\right) \Theta\left(z_{j}-z_{i}\right) \ldots \Theta\left(z_{n}-z_{n-1}\right) \times \\
e^{i\left(\Delta_{0}-\Delta_{j}-\ldots \Delta_{n}\right)\left(z_{i}-z_{1}\right)} e^{i \Delta_{j}\left(z_{j}-z_{1}\right)} \ldots e^{i \Delta_{n}\left(z_{n}-z_{1}\right)} e^{-i \Delta_{0}\left(z_{i}+z_{j}+\ldots z_{n}-n \times z_{1}\right)} .
\end{array}
$$

The sum in eq.(36) goes over all permutations between $i, j, \ldots n$. We would like to draw attention that the contribution of diagrams where ejected nucleon interacts with say nucleon "2" then with nucleon "3" and then again with nucleon "2" is exactly zero. In coordinate representation this follows from the structure of the product of $\Theta$-functions. In the momentum representation this follows from the possibility to close the contour of integration in the complex plane without encountering nucleon poles (see discussion in Section II.C of Ref. [38]).

It is easy to check that in the case of small excitation energies i.e. $\left(\Delta_{0}, \Delta_{i}, \Delta_{j} \ldots \Delta_{n} \rightarrow 0\right)$ :

$$
\left.\mathcal{O}^{(n)}\left(z_{1}, z_{i}, z_{j}, . . z_{n}, \Delta_{0}, \Delta_{i}, \Delta_{j}, \ldots \Delta_{n}\right)\right|_{\Delta_{0}, \Delta_{i, k, n} \rightarrow 0} \Rightarrow \Theta\left(z_{i}-z_{1}\right) \Theta\left(z_{j}-z_{1}\right) \ldots \Theta\left(z_{n}-z_{1}\right),
$$

and eqs.(34,35) are reduced to the conventional form of the Glauber approximation, with a simple product of the $\Theta$-functions. Within this particular approximation the sum over all $n$-fold rescattering amplitudes can be represented in the form of optical potential.

However, usually in high-energy $\left(e, e^{\prime} N\right)$ reaction the excitation energies are not too small. The use of the $\mathcal{O}^{(n)}\left(z_{1}, z_{i}, z_{j}, \ldots z_{n}, \Delta_{0}, \Delta_{i}, \Delta_{j}, \ldots \Delta_{n}\right)$, defined according to eq.(36) instead of simple product of $\Theta$ functions is the generalization of nonrelativistic Glauber approximation to the processes where comparatively large excitation energies are important. The practical consequence of the difference between $\mathcal{O}^{(n)}$ and usual $\Theta$ functions is that for sufficiently large excitation energies the sum of $n$-fold rescatterings differs substantially from the simple optical model limit.

To illustrate the deviations from the conventional Glauber approximation (which is expressed by using a simple product of the $\Theta$ functions) in Fig.4 we compare $\mathcal{O}^{(2)}\left(z_{1}, z_{2}, z_{3}, \Delta_{0}, \Delta_{1}, \Delta_{2}\right)$ function with $\Theta\left(z_{2}-z_{1}\right) \Theta\left(z_{3}-z_{1}\right)$ for $\left(e, e^{\prime} p\right)$ scattering off ${ }^{3} H e$ target. We use the kinematics for three body breakup in the final state. Figure demonstrates a considerable deviation between $\mathcal{O}^{(2)}$ and the product of $\Theta$-functions already at comparatively low excitation energies. For example, the real parts differ by more than $20 \%$ already for $\sim 60 \mathrm{MeV}$, leading to comparable difference of the double rescattering amplitude calculated including effects of longitudinal momentum transfer. The detailed numerical studies of these effects will be presented elsewhere. 

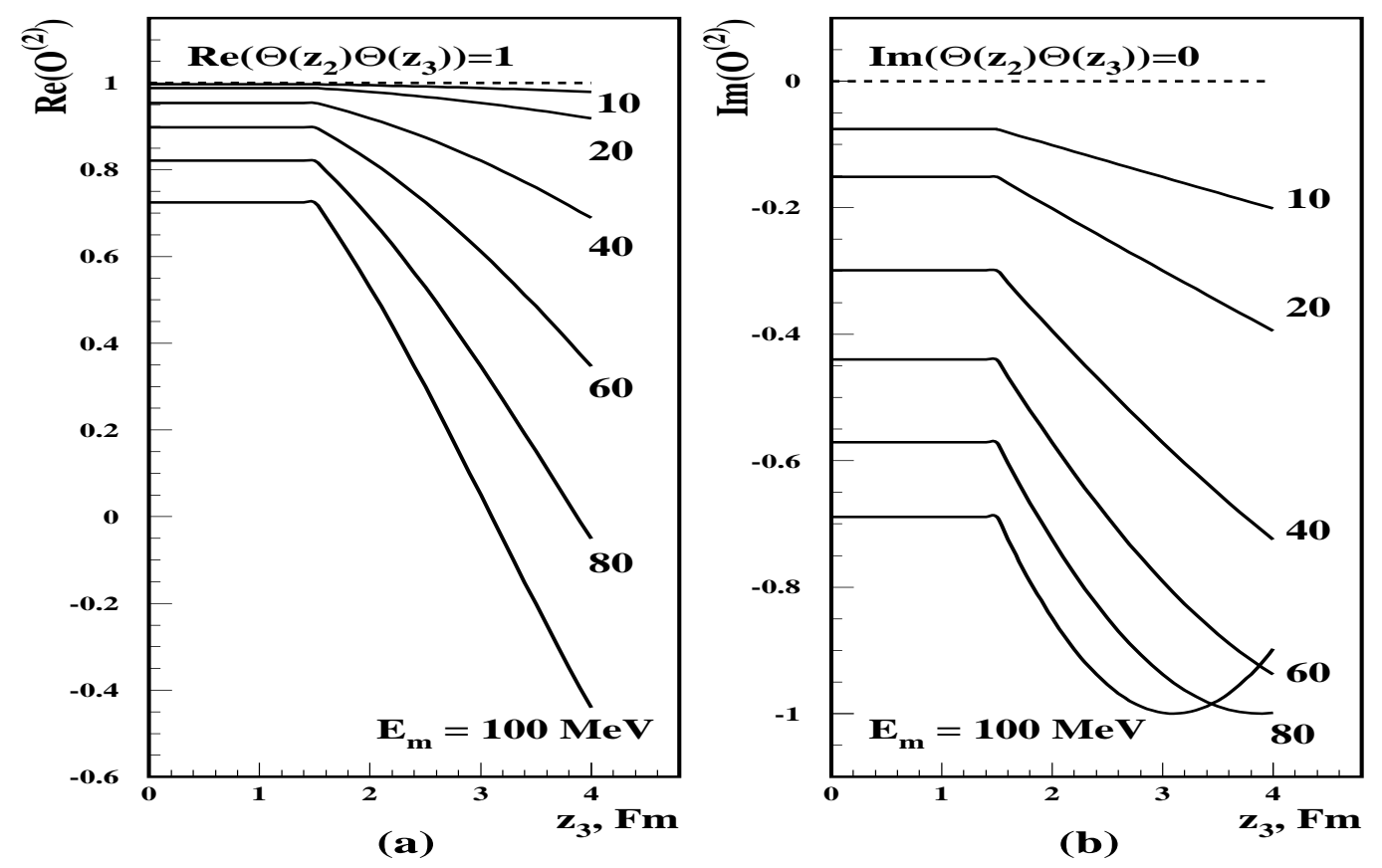

Fig. 4 Dependence of $\mathcal{O}^{(2)}\left(z_{1}, z_{2}, z_{3}, \Delta_{0}, \Delta_{1}, \Delta_{2}\right)$ and $\Theta\left(z_{2}-z_{1}\right) \Theta\left(z_{3}-z_{1}\right)$ on $z_{3}$ for different values of missing energy $E_{m}$ for $z_{1}=0, z_{2}=1.5 \mathrm{Fm}$ and $\Delta_{1}=\Delta_{2}=0$. a) Comparison of $\operatorname{Re} \mathcal{O}^{(2)}(\ldots)$ (solid line) with $\operatorname{Re} \Theta\left(z_{2}-z_{1}\right) \Theta\left(z_{3}-z_{1}\right)=1$ (dashed line) and b) comparison of $\operatorname{Im} \mathcal{O}^{(2)}(\ldots)$ (solid line) with $\operatorname{Im} \Theta\left(z_{2}-z_{1}\right) \Theta\left(z_{3}-z_{1}\right)=0$ (dashed line).

Thus we conclude, that the conventional Glauber approximation which neglects nuclear Fermi motion is applicable in the case of small values of the residual nucleus excitation energies only.

\section{FSI AND THE STUDY OF SHORT-RANGE NUCLEON CORRELATIONS IN NUCLEI}

It is generally believed that experimental condition $\left|\vec{p}_{m}\right|=\left|\vec{p}_{f}-\vec{q}\right|>k_{F}$, (where $k_{F} \sim$ $250 \mathrm{MeV} / \mathrm{c}$ is momentum of Fermi surface for a given nucleus) will enhance the contribution to the cross section from the short-range nucleon correlations in the nucleus wave function. However simple impulse approximation relation (eq.(6i)) is, in general, distorted by the FSI. Let us denote the internal momentum of the knock-out nucleons prior to the collision as $\vec{p}_{1}\left(p_{1 z}, p_{1 t}\right)$. It follows from eqs.(19),(30):

$$
\vec{p}_{1 t}=\vec{p}_{m t}-\vec{k}_{t},
$$

where $\vec{p}_{m t}$ the transverse component of the measured missing momentum, and $k_{t}$ is the momentum transferred in rescattering. Average $<\vec{k}_{t}^{2}>\sim 0.1 \mathrm{GeV}^{2}$ in the integral over $k_{t}$ are determined by the slope of the $N N$ amplitude. The longitudinal component of the 
nucleon momentum in the initial state can be evaluated through its value in the pole of the rescattered nucleon propagator (see e.g. eqs.(17),(29)):

$$
p_{1 z}=p_{m}^{z}+\Delta_{0}
$$

where $p_{m}^{z}$ is longitudinal component of the measured missing momentum and $\Delta_{0}$ represents the excitation energy of the residual nuclear system (see eq.(15)). $\Delta_{0}$ is always positive (eq.(16)). Thus, if measured $p_{z m}>k_{F}$ then, $p_{1 z}$ is even larger, i.e. $\left(p_{z 1}>p_{z m}\right)$ and therefore the FSI amplitude is as sensitive to the short range correlations as the IA amplitude. In particular, within the approximation when high-momentum component of the nuclear spectral function is due to two-nucleon short-range correlations [30]39] the condition $p_{z m}>$ $k_{F}$ corresponds to projectile electron scattering off the forward moving nucleon of the twonucleon correlation accompanied by the emission of backward nucleon.

Situation is opposite if measured momentum $p_{z m}<-k_{F}$. It follows from eq.(39), that in this case the momenta in the wave function contributing to rescattering amplitude are smaller than those for IA: $\left|p_{1}^{z}\right|=\left|p_{m}^{z}\right|-\Delta_{0}<\left|p_{m}^{z}\right|$.

Experimentally, this situation corresponds to the forward nucleon electroproduction at $\frac{Q^{2}}{2 m q_{0}} \equiv x>1$. However an important feature in this case is that in exclusive electroproduction the value of $\Delta_{0}$ is measured experimentally and can be easily chosen so that momenta entering in the ground state wave function would be larger than $k_{F}$. Therefore to investigate the short-range correlations in the $\left(e, e^{\prime} p\right)$ reactions for $x>1$, we have to impose an additional condition:

$$
\left|p_{m}^{z}\right|-\Delta_{0}>k_{F}
$$

to suppress the contribution from large internucleon distances.

Overall, we observe that in order to study short-range nucleon correlations in $\left(e, e^{\prime} N\right)$ reactions off nuclei with minimal distortions due the FSI effects it is advantageous to use the $x<1$ kinematics especially with detection of a backward going nucleon.

Above results have a simple explanation in terms of the light-cone dynamics of highenergy scattering processes. Indeed, according to eq.(16) $\Delta_{0}$ does not disappear with increase of energy. Hence the non-conservation of the longitudinal momentum of nucleons given by eq.(39) : $p_{1 z}-p_{m}^{z}=\Delta_{0}$ remains finite in the high-energy limit. However, the rescattering of an energetic knock-out nucleon practically does not change the "-" component of its four-momentum $p_{-} \equiv E-p_{z},\left(p_{-}\right.$is the longitudinal momentum as defined in light-cone variables, where $p^{\mu} \equiv p^{\mu}\left(p_{+}, p_{-}, p_{t}\right)$ with $\left.p_{ \pm}=E \pm p_{z}\right)$. Really, if we define $p_{1-}=m-p_{1 z}$ and $p_{m-}=p_{f-}-q_{-}=m-E_{m}-p_{m z}$, where $E_{m}=m+E_{A-1}-M_{A}$ is the missing energy, then according to eq.(39) the non-conservation of "-" component is:

$$
p_{1-}-p_{m-} \approx \frac{Q^{2}}{2 q^{2}} E_{m}=\frac{E_{m}}{2\left(1+\frac{q_{0}}{2 m x}\right)} .
$$

It vanishes with increase of the virtual photon energy $q_{0}$. Hence, the physical interpretation of eq.(39) is that at high energies elastic FSI does not change noticeably the light-cone "-." component of the struck nucleon momentum. This reasoning indicates that description of the FSI in high-energy processes should be simplified when treated within the framework of 
the light-cone dynamics. Our previous analysis of $x>1$, large $Q^{2}$ data on inclusive $\left(e, e^{\prime}\right)$ processes is consistent with this idea 40.

The above observation helps to rewrite the deduced formulae in the form accounting for, in the straightforward way, that high-energy processes develop along the light-cone.

Let us introduce light-cone momenta $\alpha_{i} \equiv A \frac{p_{i-}}{P_{A-}}$. Here $\alpha / A$ is a momentum fraction of target nucleus carried by the nucleon- $i$. Using the above discussed expressions for $p_{m-}$ and $p_{1-}$ and eqs.(15,16) for the propagator of a fast nucleon we obtain:

$$
\frac{1}{\left[p_{z}^{m}+\Delta_{0}-p_{1 z}+i \epsilon\right]}=\frac{1}{m\left[\alpha_{1}-\alpha_{m}+\frac{q_{0}-q}{q m} E_{m}+i \epsilon\right]} \approx \frac{1}{m\left[\alpha_{1}-\alpha_{m}-\frac{Q^{2}}{2 q^{2}} \frac{E_{m}}{m}+i \epsilon\right]}
$$

In the kinematics where relativistic effects in the wave function of the target and residual nucleus are small and $\alpha_{j} \approx 1-\frac{p_{j z}}{m}$, there is a smooth correspondence between nonrelativistic and light-cone wave functions of the nucleus [30], i.e. $\phi_{A}\left(p_{1}, \ldots p_{j}, . . p_{A}\right) \approx$ $\phi_{A}\left(\alpha_{1}, p_{1 t}, \ldots \alpha_{j}, p_{j t}, \ldots \alpha_{A}, p_{A}\right) / m^{\frac{A}{2}}$. Therefore the amplitude of single rescattering - eq.(17) can be rewritten as:

$$
\begin{array}{r}
T^{(b)}=-\frac{\sqrt{(2 \pi)^{3}}(2 \pi)^{3}}{2 m} \int \psi_{A}\left(\alpha_{1}, p_{1 t}, \alpha_{2}, p_{2 t}, \alpha_{3}, p_{3 t}\right) F_{1}^{e m}\left(Q^{2}\right) \frac{f^{N N}}{\left[\alpha_{1}-\alpha_{m}-\frac{Q^{2}}{2 q^{2}} \frac{E_{m}}{m}+i \epsilon\right]} \\
\psi_{A-1}\left(\alpha_{2}^{\prime}, p_{2 t}^{\prime}, \alpha_{3}, p_{3 t}\right) \frac{d \alpha_{1} d^{2} p_{1 t}}{(2 \pi)^{3}} \frac{d \alpha_{3} d^{2} p_{3 t}}{(2 \pi)^{3}} .
\end{array}
$$

where according to eq.(11) $\alpha_{2}=\alpha_{2}^{\prime}=3-\alpha_{1}-\alpha_{3}$. Eq.(43) shows that in the limit when $\frac{Q^{2}}{2 q^{2}} \frac{E_{m}}{m} \rightarrow 0$, the amplitude $T^{(b)}$ is expressed through the light-cone variables and the lightcone wave functions of nucleus. Note that the eikonal scattering corresponds to the linear, in $\alpha_{1}$, propagator of the fast nucleon. It is instructive that regime of the light-cone dynamics is reached in eq.(43) at relatively moderate energies. Indeed let as consider kinematics when $\alpha_{1}$ is close to unity, (which is the case in our analysis). At $q_{0} \sim 2 \mathrm{GeV}, \frac{Q^{2}}{2 q^{2}} \frac{E_{m}}{m}=\frac{1}{2\left(1+\frac{q_{0}}{2 m x}\right)} \frac{E_{m}}{m} \sim$ $(0.05-0.07) \ll 1$. For estimate we take $x=1$ and for missing energy $E_{m} \sim 0.2-0.3 \mathrm{GeV}$ which is close to the limit of applicability of the description of nuclei as a many-nucleon system (cf. [31]). Similar reasoning is applicable for the double rescattering amplitude in eq.(29). Here we obtain:

$$
\begin{aligned}
T^{(d)=} & \frac{\sqrt{(2 \pi)^{3}}(2 \pi)^{3}}{4 m^{2}} \int \psi_{A}\left(\alpha_{1}, p_{1 t}, \alpha_{2}, p_{2 t}, \alpha_{3}, p_{3 t}\right) F_{1}^{e m}\left(Q^{2}\right) \times \\
& \frac{f^{N N}\left(p_{1 t}-p_{m t}-\left(p_{3 t}^{\prime}-p_{3 t}\right)\right)}{\left[\alpha_{1}-\alpha_{m}-\frac{Q^{2}}{2 q^{2}} \frac{E_{m}}{m}+i \epsilon\right]} \frac{f^{N N}\left(p_{3 t}^{\prime}-p_{3 t}\right)}{\left[\alpha_{3}-\alpha_{3}^{\prime}-\frac{Q^{2}}{2 q^{2}} \frac{k_{3 t}^{2}}{2 m^{2}}+i \epsilon\right]} \psi_{A-1}\left(\alpha_{2}, p_{2 t}^{\prime}, \alpha_{3}, p_{3 t}^{\prime}\right) \\
& \frac{d \alpha d^{2} p_{1 t}}{(2 \pi)^{3}} \frac{d \alpha_{3} d^{2} p_{3 t}}{(2 \pi)^{3}} \frac{d \alpha_{3}^{\prime} d^{2} p_{3 t}^{\prime}}{(2 \pi)^{3}} .
\end{aligned}
$$

Another interesting consequence of the representation of the scattering amplitude through the light-cone variables, is the simple form of the closure approximation for the sum over the residual $(A-1)$ nuclear states in $A\left(e, e^{\prime} N\right)(A-1)$ reaction. When summing over $E_{m}$ at fixed $p_{m}$ the rescattering amplitudes (cf. eq.(17)) could not be factored out from the sum because they depend on $E_{m}$ through the $\Delta$ factors (cf. eq.(16)). In the case 
of the light-cone representation (cf. eq.(43)) the analogous procedure [31] is to sum over $p_{+} \approx m+E_{m}+p_{m z}$ at fixed $\alpha_{m}$. It follows from eqs.44) and (45) that in such a sum the scattering amplitude is independent of $p_{+}$and therefore the application of closure has a simple form.

Note that the present discussion of the light-cone dynamics is by no means complete, since we don't consider the relativistic effects which enter into the nuclear wave functions. The extension of the current analysis to the light-cone formalism will be presented elsewhere.

\section{CONCLUSIONS}

The Feynman diagram approach to the calculation of final state interactions at high energy $\left(e, e^{\prime} N\right)$ reactions off nuclei provides a natural framework for the generalization of the conventional nonrelativistic Glauber approximation to high-energy processes. This approach adequately describes also the light-cone dynamics characteristic at high-energy reactions.

It follows from the consideration of Feynman diagrams that the formulae of the conventional Glauber approximation are a legitimate approximation for sufficiently small values of residual nuclear system's excitation energy (missing energy). Beyond this kinematic region, conventional approximation should be modified to describe correctly relativistic kinematics and the dynamics of FSI. This can be done within the generalized eikonal approach which is developed in the paper for $\left(e, e^{\prime} N\right)$ knock-out reactions.

The obtained formulae allow to find a kinematic domain preferable for the investigation of short-range nucleon correlations in nuclei. We demonstrate that scattering off forward moving nucleons (which corresponds to production of backward going nucleon spectators from destruction of short-range pair correlations [30]) is preferable for the investigation of short-range nucleon correlations in nuclei. We found an additional kinematic condition: $\left|p_{m}^{z}\right|-\Delta_{0}>k_{F}$ for semi-exclusive reactions to enhance the contribution of short-range nucleon correlations at $x>1$ and reduce FSI.

We demonstrate also that dominance of light-cone dynamics follows directly from the analysis of the Feynman diagrams, and that the " -" component of the target nucleon momentum is almost conserved in FSI. Therefore, by measuring the "-" component of the missing momenta we directly tag the preexisting momenta in the light cone nuclear wave function.

\section{ACKNOWLEDGMENTS}

We would like to thank G. A. Miller for useful suggestions and comments. The work was supported in part by the Israel-USA Binational Science Foundation Grant No. 92000126 and by the U.S. Department of Energy under Contract No. DE-FG02-93ER40771. 


\section{APPENDIX A: WHY CLOSURE APPROXIMATION IN GENERAL IS APPLICABLE IN LIGHT-CONE BUT NOT IN THE NUCLEUS REST FRAME}

In the calculation of $n$-fold rescattering amplitude of Fig.1 we assumed the decoupling (from the excitation energies of intermediate states) of the propagator of high energy knocked-out nucleon $-D\left(p_{1}+q\right)^{-1}$. Such a decoupling allows to use the closure over the sum over the excitations of intermediate nuclear states. As a result the scattering amplitude in eq.(11), is calculated in terms of the propagators of free spectator nucleons in the intermediate states.

To visualize the conditions when the decoupling of high-energy part of the diagram of Fig.1 from low-energy part would be valid we consider two reference frame descriptions: Nucleus rest frame (Lab frame) and Light Cone.

In the Lab frame the inverse propagator of energetic knocked-out nucleon: $-D\left(p_{1}+q\right)=$ $\left(p_{1}+q\right)^{2}-m^{2}+i \epsilon$ can be written as:

$$
\left(p_{1}+q\right)^{2}-m^{2}=p_{1}^{2}+2 E_{1} q_{0}-2 \vec{p}_{1} \cdot \vec{q}+q^{2}-m^{2}=2|\vec{q}|\left[\frac{p_{1}^{2}-m^{2}}{2|\vec{q}|}+E_{1} \frac{q_{0}}{|\vec{q}|}-p_{1 z}-\frac{Q^{2}}{|\overrightarrow{2} q|}\right]
$$

It follows from the right hand side of eq.(A1) that only the term $E_{1} \frac{q_{0}}{|\vec{q}|}-p_{1 z}$ survives in limit of large momentum transfer $(\vec{q})$ and fixed $x_{B j}$. Thus in high energy limit within Lab frame description one should retain the dependence of propagator on the excitation energy of intermediate state (via $E_{1}$ ). Therefore, unless the $E_{1}$ dependence of the propagator of knocked-out nucleon can be neglected the use of closure over the intermediate nuclear states can not be justified. In the Lab frame description such a neglection is legitimate in the nonrelativistic limit only where the term $\frac{p_{1}^{2}}{2 m^{2}} \ll 1$ is neglected everywhere in the expression of the scattering amplitude. Such a restriction on the applicability of the closure for the sum over the intermediate states is of crucial importance for the models where relativistic effects are treated on the basis of the Lab frame description.

Above calculation does not take into account additional approximate conservation law characteristic for light-cone dynamics. Let us introduce light-cone momenta for four-vectors as: $p^{\mu}\left(p_{+}, p_{-}, p_{t}\right)$, where $p_{ \pm}=E \pm p_{z}$. Using these definitions, for the inverse propagator of knocked-out nucleon one obtains the form:

$$
\left(p_{1}+q\right)^{2}-m^{2}=p_{1}^{2}+p_{1+} q_{-}+p_{1-} q_{+}+q^{2}-m^{2}=q_{+}\left[\frac{p_{1}^{2}-m^{2}}{q_{+}}+p_{1+} \frac{q_{-}}{q_{+}}+p_{1-}-\frac{Q^{2}}{q_{+}}\right] \text {. }
$$

As follows from the above equation that only term, that survives at fixed $x_{B j}$ and high energy transfer limit, is $p_{1-}$. Therefore at fixed $p_{-}$we found effective factorization of high-energy propagator from low energy intermediate nuclear part whose excitation energy on light cone is defined by the $p_{1+} 30,31$. Such a decoupling applies for any values of Fermi momenta of the target nucleon (no restriction like $\frac{p_{1}^{2}}{2 m^{2}} \ll 1$ is needed). Therefore it is possible to extend the applicability of the closure over intermediate states of the residual nucleus to the domain of relativistic momenta of target nucleons. The price is to introduce the light-cone wave function's of the target (similar to the case of pQCD). 
Note that the considerations in present work are restricted by small Fermi momenta (eq.(4)) since we use $\frac{p_{1}^{2}}{2 m^{2}} \ll 1$ in the scattering amplitude. For larger Fermi momenta a legitimate way to generalize obtained results is to use light-cone description, which is out of scope of the present paper. Note that light-cone mechanics of nuclei is rather similar to the nonrelativistic ones 30,32.

\section{APPENDIX B: COORDINATE SPACE REPRESENTATION OF THE SCATTERING AMPLITUDE}

\section{Single scattering amplitude}

We now will transform the single scattering amplitude of eq.(17) to coordinate space. Inserting the configuration space representation of ground state and residual state wave functions according to eq.(17) into eq.(17) and using energy-momentum conservation of eq.(11), for $T^{(b)}$ we obtain:

$$
\begin{aligned}
T^{(b)}= & -\frac{1}{2} \int d^{3} x_{1} d^{3} x_{2} d^{3} x_{3} d^{3} y_{2} d^{3} y_{3} e^{-i\left(\vec{x}_{1}-\vec{x}_{2}\right) \cdot \vec{p}_{1}} \phi_{A}\left(x_{1}, x_{2}, x_{3}\right) \frac{F_{1}^{e m}\left(Q^{2}\right) f^{N N}\left(p_{2 t}^{\prime}-p_{2 t}\right)}{\left[p_{z}^{m}+\Delta_{0}-p_{1 z}+i \epsilon\right]} \\
& e^{i \vec{y}_{2} \cdot \vec{p}_{A-1}} e^{-i \vec{p}_{3} \cdot\left(\left(\vec{x}_{3}-\vec{x}_{2}\right)-\left(\vec{y}_{3}-\vec{y}_{2}\right)\right)} \phi_{A-1}^{\dagger}\left(y_{2}, y_{3}\right) \frac{d^{3} p_{1}}{(2 \pi)^{3}} \frac{d^{3} p_{3}}{(2 \pi)^{3}} \\
= & -\frac{1}{2} \int d^{3} x_{1} d^{3} x_{2} d^{3} x_{3} d^{3} y_{2} d^{3} y_{3} e^{-i\left(\vec{x}_{1}-\vec{x}_{2}\right) \cdot \vec{p}_{1}} \phi_{A}\left(x_{1}, x_{2}, x_{3}\right) \frac{F_{1}^{e m}\left(Q^{2}\right) f^{N N}\left(p_{2 t}^{\prime}-p_{2 t}\right)}{\left[p_{z}^{m}+\Delta_{0}-p_{1 z}+i \epsilon\right]} \\
& e^{i \vec{y}_{2} \cdot \vec{p}_{A-1}} \delta^{3}\left(\left(x_{3}-x_{2}\right)-\left(y_{3}-y_{2}\right)\right) \phi_{A-1}^{\dagger}\left(y_{2}, y_{3}\right) \frac{d^{3} p_{1}}{(2 \pi)^{3}} .
\end{aligned}
$$

Next, we introduce relative and CM coordinates as:

$$
y_{2}=\frac{y_{23}}{2}+y_{c m} ; \quad y_{3}=\frac{y_{23}}{2}-y_{c m}
$$

and separate internal and CM motion of the recoil pn system:

$$
\phi_{A-1}\left(y_{2}, y_{3}\right)=\phi\left(y_{23}\right) e^{i y_{c m}\left(p_{p}+p_{n}\right)} .
$$

As a result, $T^{(b)}$ takes the form:

$$
\begin{aligned}
T^{(b)}=-\frac{1}{2} \int & d^{3} x_{1} d^{3} x_{2} d^{3} x_{3} d^{3} y_{2} d^{3} y_{3} e^{-i\left(\vec{x}_{1}-\vec{x}_{2}\right) \cdot \vec{p}_{1}} \phi_{A}\left(x_{1}, x_{2}, x_{3}\right) \frac{F_{1}^{e m}\left(Q^{2}\right) f^{N N}\left(p_{2 t}^{\prime}-p_{2 t}\right)}{\left[p_{z}^{m}+\Delta_{0}-p_{1 z}+i \epsilon\right]} \\
& e^{-i \frac{\left(\vec{x}_{2}-\vec{x}_{3}\right)}{2} \cdot \vec{p}_{m}} \phi^{\dagger}\left(x_{2}-x_{3}\right) \frac{d^{3} p_{1}}{(2 \pi)^{3}} .
\end{aligned}
$$

To integrate over $p_{1 z}$ we use the coordinate space representation of the nucleon propagator $\frac{1}{\left[p_{z}^{m}+\Delta_{0}-p_{1 z}+i \epsilon\right]}$ according to eq.(18). Inserting eq.(18) in eq.(B4), one can integrate over $p_{1 z}$ : $\int \exp \left(-i p_{1 z}\left(z_{1}-z_{2}+z^{0}\right)\right) d p_{1 z}=2 \pi \delta\left(z_{1}-z_{2}+z^{0}\right)$. After integrating over $d z^{0}$ we obtain: 


$$
\begin{aligned}
T^{(b)}= & \frac{i}{2} \int d^{3} x_{1} d^{3} x_{2} d^{3} x_{3} e^{i\left(\vec{b}_{2}-\vec{b}_{1}\right) \cdot\left(\vec{p}_{1}^{t}-\vec{p}_{m}^{t}\right)} \phi_{A}\left(x_{1}, x_{2}, x_{3}\right) F_{1}^{e m}\left(Q^{2}\right) \\
& f^{N N}\left(p_{2 t}^{\prime}-p_{2 t}\right) \Theta\left(z_{2}-z_{1}\right) e^{-i \frac{3}{2} \vec{p}_{m} \cdot \vec{x}_{1}} e^{i \Delta_{0}\left(z_{2}-z_{1}\right)} \phi^{\dagger}\left(x_{2}-x_{3}\right) \frac{d^{2} p_{1}^{t}}{(2 \pi)^{2}} \\
= & \frac{i}{2} \int d^{3} x_{1} d^{3} x_{2} d^{3} x_{3} \phi_{A}\left(x_{1}, x_{2}, x_{3}\right) \Theta\left(z_{2}-z_{1}\right) e^{i\left(\vec{b}_{2}-\vec{b}_{1}\right) \cdot \vec{k}_{t}} F_{1}^{e m}\left(Q^{2}\right) \\
& f^{N N}\left(p_{2 t}^{\prime}-p_{2 t}\right) e^{i \Delta_{0}\left(z_{2}-z_{1}\right)} e^{-i \frac{3}{2} \vec{p}_{m} \cdot \vec{x}_{1}} \phi^{\dagger}\left(x_{2}-x_{3}\right) \frac{d^{2} k}{(2 \pi)^{2}}
\end{aligned}
$$

where we define the momentum transferred in the rescattering as $\vec{k}_{t}=\vec{p}^{t_{1}}-\vec{p}^{t_{m}}=\vec{p}^{\prime}{ }_{2 t}-\vec{p}_{2 t}$ and $\vec{b}_{1}, \vec{b}_{2}$ are transverse components of vectors $\vec{x}_{1}, \vec{x}_{2}$.

\section{Double scattering amplitude}

Integration in eq.(29) can be performed in the coordinate space, using the Fourier transform of the wave functions according to eq.(7) and by introducing the $\vec{L}$ and $\vec{k}_{3}$ :

$$
\vec{L}=\frac{\vec{p}_{3}{ }^{\prime}+\vec{p}_{3}}{2} ; \quad \vec{k}_{3}=\vec{p}_{3}{ }^{\prime}-\vec{p}_{3}
$$

Then for $T^{(d)}$ we obtain:

$$
\begin{aligned}
T^{(d)}= & \frac{1}{4} \int d^{3} x_{1} d^{3} x_{2} d^{3} x_{3} d^{3} y_{2} d^{3} y_{3} e^{-i\left(\vec{x}_{1}-\vec{x}_{2}\right) \cdot \vec{p}_{1}} \phi_{A}\left(x_{1}, x_{2}, x_{3}\right) F_{1}^{e m}\left(Q^{2}\right) \\
& \frac{f^{N N}\left(p_{2}^{\prime}-p_{2}\right)}{\left[p_{z}^{m}+\Delta_{0}-p_{1 z}+i \epsilon\right]} \frac{f^{N N}\left(p_{3}^{\prime}-p_{3}\right)}{\left[\Delta_{3}-\left(k_{3 z}\right)+i \epsilon\right]} \\
& e^{i \vec{y}_{2} \cdot \vec{p}_{A-1}} e^{-i \frac{\vec{k}_{3}}{2} \cdot\left[\left(\vec{x}_{2}-\vec{x}_{3}\right)+\left(\vec{y}_{2}-\vec{y}_{3}\right)\right]} e^{i \vec{L} \cdot\left[\left(\vec{x}_{2}-\vec{x}_{3}\right)-\left(\vec{y}_{2}-\vec{y}_{3}\right)\right]} \phi_{A-1}^{\dagger}\left(y_{2}, y_{3}\right) \frac{d^{3} p_{1}}{(2 \pi)^{3}} \frac{d^{3} L}{(2 \pi)^{3}} \frac{d^{3} k_{3}}{(2 \pi)^{3}} .
\end{aligned}
$$

Since we consider soft rescatterings of a high energy (knocked-out) nucleon off a slow spectator, we can use the observation that the scattering amplitude for two-body scattering $f^{N N}\left(p_{3}^{\prime}-p_{3}\right)$ depends mainly on transverse components of transferred momentum $\vec{k}_{3 t}$ and is practically independent of $\vec{L}$. Therefore we can perform integration over $d^{3} L$ invoking the factor $\delta^{3}\left(x_{2}-x_{3}-\left(y_{2}-y_{3}\right)\right)$. Similar to the previous section using eqs.(B2) and (B3) allows to perform the integration over $d^{3} y_{c m} d^{3} y_{23}$ :

$$
\begin{aligned}
T^{(d)}= & \frac{1}{4} \int d^{3} x_{1} d^{3} x_{2} d^{3} x_{3} e^{-i\left(\vec{x}_{1}-\vec{x}_{2}\right) \cdot \vec{p}_{1}} e^{-i \vec{k}_{3} \cdot\left(\vec{x}_{2}-\vec{x}_{3}\right)} \phi_{A}\left(x_{1}, x_{2}, x_{3}\right) F_{1}^{e m}\left(Q^{2}\right) \\
& \frac{f^{N N}}{\left[p_{z}^{m}+\Delta_{0}-p_{1 z}+i \epsilon\right]} \frac{f^{N N}}{\left[\Delta_{3}-\left(k_{3 z}\right)+i \epsilon\right]} e^{-i \frac{\vec{x}_{2}-\vec{x}_{3}}{2} \vec{p}_{m}} \phi^{\dagger}\left(x_{2}-x_{3}\right) \frac{d^{3} p_{1}}{(2 \pi)^{3}} \frac{d^{3} k_{3}}{(2 \pi)^{3}} .
\end{aligned}
$$

Furthermore we can take the integral over $p_{1 z}$ similarly to the case of single rescattering amplitude, using the eq.(18). The integration by $k_{3 z}$ can be done using the representation: $\frac{1}{\Delta_{3}-k_{3 z}+i \epsilon}=-i \int \Theta\left(z^{k_{3}}\right) \exp i\left(\Delta_{3}-k_{3 z}\right) z^{k_{3}} d z^{k_{3}}$. The integration over $d p_{1 z}$ and $d k_{3 z}$ leads to the factor: $2 \pi \delta\left(z^{0}-\left(z_{2}-z_{1}\right)\right)$ and $2 \pi \delta\left(z^{k_{3}}-\left(z_{3}-z_{2}\right)\right)$ respectively. After performing integration over $d z^{0}$ and $d z^{k_{3}}$ and defining $\vec{k}_{1}=\vec{p}_{1}^{t}-\vec{p}_{m}^{t}$ we obtain: 


$$
\begin{aligned}
T^{(d)} & =\frac{i^{2}}{4} \int d^{3} x_{1} d^{3} x_{2} d^{3} x_{3} \phi_{A}\left(x_{1}, x_{2}, x_{3}\right) F_{1}^{e m}\left(Q^{2}\right) \\
& \times\left[\Theta\left(z_{2}-z_{1}\right) f^{N N}\left(k_{1 t}-k_{3 t}\right) e^{i \vec{k}_{1 t} \cdot\left(\vec{b}_{2}-\vec{b}_{1}\right)} e^{i \Delta_{0}\left(z_{2}-z_{1}\right)} \frac{d^{2} k_{1}}{(2 \pi)^{2}}\right] \\
& \times\left[\Theta\left(z_{3}-z_{2}\right) f^{N N}\left(k_{3 t}\right) e^{i \vec{k}_{3 t} \cdot\left(\vec{b}_{3}-\vec{b}_{2}\right)} e^{i \Delta_{3}\left(z_{3}-z_{2}\right)} \frac{d^{2} k_{3}}{(2 \pi)^{2}}\right] e^{-i \frac{3}{2} \vec{x}_{1} \cdot \vec{p}_{m}} \phi^{\dagger}\left(x_{2}-x_{3}\right) \\
& =\frac{i^{2}}{4} \int d^{3} x_{1} d^{3} x_{2} d^{3} x_{3} \phi_{A}\left(x_{1}, x_{2}, x_{3}\right) F_{1}^{e m}\left(Q^{2}\right) \\
& \times\left[\Theta\left(z_{2}-z_{1}\right) f^{N N}\left(k_{2 t}\right) e^{i \vec{k}_{2 t} \cdot\left(\vec{b}_{2}-\vec{b}_{1}\right)} e^{i\left(\Delta_{0}-\Delta_{3}\right)\left(z_{2}-z_{1}\right)} \frac{d^{2} k_{2}}{(2 \pi)^{2}}\right] \\
& \times\left[\Theta\left(z_{3}-z_{2}\right) f^{N N}\left(k_{3 t}\right) e^{i \vec{k}_{3 t} \cdot\left(\vec{b}_{3}-\vec{b}_{1}\right)} e^{i \Delta_{3}\left(z_{3}-z_{1}\right)} \frac{d^{2} k_{3}}{(2 \pi)^{2}}\right] e^{-i \frac{3}{2} \vec{x}_{1} \cdot \vec{p}_{m}} \phi^{\dagger}\left(x_{2}-x_{3}\right),
\end{aligned}
$$

where at the last step we do the replacement $\vec{k}_{1 t} \rightarrow \vec{k}_{2 t}+\vec{k}_{3 t}$. 


\section{REFERENCES}

[1] CEBAF - Conceptual design report, Southeastern Universities Research Association, Newport News, April 1990.

[2] Technical Report of the HERMES Experiment, DESY, July 1991.

[3] The ELFE project, edited by J. Arvieux and E. De Sanctis (Italian Physical Society, Bologna, 1993); J. Arvieux and B. Pire, Progress in nuclear and Particle Physics 30, 299 (1995).

[4] C. D. Epp and T. A. Griffy, Phys. Rev. C1, 1633, (1970); F. Cannata, J. P. Dedonder and F. Lenz, Ann. Phys. (N.Y.) 143, 84 (1982).

[5] R. J. Glauber Phys. Rev. 100, 242 (1955); Lectures in Theoretical Physics, v.1, ed. W. Brittain and L. G. Dunham, Interscience Publ., N.Y. 1959.

[6] D. R. Yennie, in Hadronic interactions of Electrons and photons, edited by J. Cummings and D. Osborn (Academic, New York, 1971), p.321.

[7] E. J. Moniz and G. D. Nixon, Ann. Phys. (N.Y.) 67, 58 (1971).

[8] G. R. Farrar, L. L. Frankfurt, H. Liu and M. I. Strikman Phys. Rev. Lett. 61, 686 (1988).

[9] T.-S. H. Lee and G. A. Miller, Phys.Rev. C 45, 1863 (1992).

[10] B. K Jennings and G. A. Miller, Phys. Lett. B318, 7 (1993).

[11] A. Kohama, K. Yazaki, and R. Seki, Nucl.Phys. A551, 687 (1993).

[12] L. L. Frankfurt, M. I. Strikman and M. Zhalov, Phys. Rev. C 50, 2189 (1994).

[13] O. Benhar et al., Phys. Rev. Lett. 69, 881 (1992).

[14] A. S. Rinat and M. F. Taragin, Phys.Rev. C52, 28 (1995).

[15] N. N. Nikolaev, A. Szczurek, et al., Phys. Lett. B317, 287 (1993).

[16] S. Frankel, W. Frati and N. R. Walet, Nucl. Phys. A580, 595 (1994).

[17] A. Bianconi, S. Boffi, D. E. Kharzeev Phys. Lett. B325, 294 (1994).

[18] E. J. Moniz, Summary Talk in PANIC-XIII, Perugia, Italy, 1993.

[19] L. L. Frankfurt, M. I. Strikman and M. Zhalov, Nucl. Phys. A515, 599 (1990).

[20] L. L. Frankfurt, E. J. Moniz, M. M. Sargsyan and M. I. Strikman, Phys. Rev. C51, 3435 (1995).

[21] A. H. Mueller, in Proceedings of Seventeenth Rencontre de Moriond, ed. J. Tran Thanh Van, Editions Frontieres, Gif-sur-Yvette, France, 1982, p.13.

[22] S. J. Brodsky in Proceedings of Thirteenth Intl. Symposium on, multiparticle Dynamics ed, W. Kittel, W. Metzegar and A. Stergiou, World Scientific, Singapore, 1982, p.963.

[23] A. Mandelstam, Nuovo Cim. 30, 1113 (1963).

[24] V. N. Gribov, JETP 29, 483 (1969).

[25] L. L. Frankfurt, G. A. Miller, and M. I. Strikman, Ann. Rev. of Nucl. and Part. Phys. 44, 501 (1994).

[26] P. Jain, B. Pire and J.P. Ralston, Phys. Rep. 271, 67 (1996).

[27] V. N. Gribov, Sov, Phys. JETP, 30, 709 (1970).

[28] L. Bertocchi, Il Nuovo Cimento, A 11, 45 (1972).

[29] L. L. Frankfurt, W. R. Greenberg, G. A. Miller, M. M. Sargsyan and M. I. Strikman, Z.Phys A352, 97, (1995).

[30] L. L. Frankfurt, M. I. Strikman, Phys. Rep. 76215 (1981).

[31] L. L. Frankfurt, M. I. Strikman, Phys. Rep. 160235 (1988). 
[32] L. L. Frankfurt, M. I. Strikman, in Modern Topics in Electron Scattering, ed. B. Frois and I. Sick (1991) p.645.

[33] B. D. Keister, W. N. Polyzou, in Advances in nuclear physics, edited J. W. Negele, E Vogt v.20, 225 (1991).

[34] L. A. Kondratyuk, J. Vogelzang and M. S. Franchenko, Phys. Lett. B98, 405 (1981).

[35] T. H. Bauer, R. D. Spital, D. R. Yennie anf F. M. Pipkin, Rev. Mod. Phys. 50, 261 (1978).

[36] S. Kuhn and K. Griffieon (spokespersons) CEBAF proposal PR-102-94, 39pp (1994).

[37] K. Egiyan, K. Griffieon and M. Strikman (spokespersons) CEBAF proposal PR-94-01994, 42pp (1995).

[38] L. Frankfurt, E. Piasetzky, M. Sargsian and M. Strikman, TAUP-2356-96, hepph/9607395, 33pp. (1996).

[39] C. Ciofi degli Atti, L. Frankfurt, S. Simula and M. Strikman, Phys. Rev. C44, 7 (1991).

[40] L. Frankfurt, M. I. Strikman , D. B. Day and M. M. Sargsyan Phys. Rev. C48, 2451 (1993). 